\title{
Authentication protocols based on low-bandwidth unspoofable channels: a comparative survey
}

\author{
L.H. Nguyen and A.W. Roscoe \\ Oxford University Computing Laboratory \\ E-mail addresses: \{Long.Nguyen, Bill.Roscoe@comlab.ox.ac.uk\}
}

\begin{abstract}
One of the main challenges in pervasive computing is how we can establish secure communication over an untrusted high-bandwidth network without any initial knowledge or a Public Key Infrastructure. An approach studied by a number of researchers is building security though human work creating a low-bandwidth empirical (or authentication) channel where the transmitted information is authentic and cannot be faked or modified. In this paper, we give an analytical survey of authentication protocols of this type. We start with non-interactive authentication schemes, and then move on to analyse a number of strategies used to build interactive pair-wise and group protocols that minimise the human work relative to the amount of security obtained as well as optimising the computation processing. In studying these protocols, we will discover that their security is underlined by the idea of commitment before knowledge, which is refined by two protocol design principles introduced in this survey.
\end{abstract}




\section{Contents}

1 Introduction $\quad 3$

2 Notation and basic definitions $\quad 5$

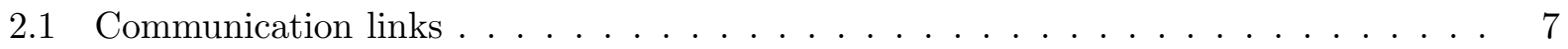

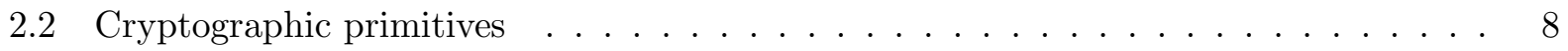

2.2.1 Commitment scheme and Commitment before knowledge . . . . . . . . . 8

2.2 .2 Short hash and digest functions . . . . . . . . . . . . . . . . . . 11

2.3 Attack model . . . . . . . . . . . . . . . . . . . . . . . . 12

2.4 Cost model . . . . . . . . . . . . . . . . . . . . . . . 13

2.4 .1 Human effort . . . . . . . . . . . . . . . . . . 13

2.4.2 Computation cost model of cryptographic primitives . . . . . . . . . . . 14

3 Non-interactive protocols $\quad 16$

3.1 Long authentication string over the empirical channel . . . . . . . . . . . 16

3.2 Short authentication strings over strong empirical channels . . . . . . . . . . . . 19

3.3 Improved version of $(\mathrm{V}-)$ MANA I . . . . . . . . . . . . . . . . . . . . 21

4 Interactive protocols $\quad 22$

4.1 Multiple empirical short authentication strings . . . . . . . . . . . 23

4.2 Indirect binding . . . . . . . . . . . . . . . . . 26

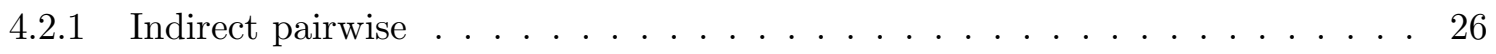

4.2 .2 Hybrid protocol . . . . . . . . . . . . . . . . . . 28

4.3 Direct binding pairwise protocols . . . . . . . . . . . . . . . . . 29

5 Group protocols $\quad 33$

5.1 Some existing direct binding group protocols $\ldots \ldots \ldots \ldots$. . . . . . . . 34

5.2 Indirect binding group protocol . . . . . . . . . . . . . . . 36

5.3 Modified versions of HCBK and SHCBK . . . . . . . . . . . . . . 37

6 Conclusions and further work $\quad 40$

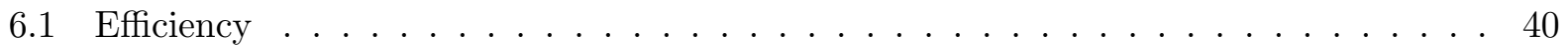

6.2 Short-term public key cryptography . . . . . . . . . . . . . . . . 41

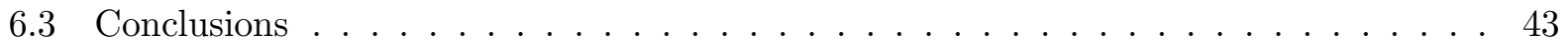

6.4 Future research . . . . . . . . . . . . . . . . . . . . . . 44

A The importance of empirical display of leader $(L, A)$ in Hybrid HCBK 48

$\begin{array}{lll}\text { B Attack on group protocol with two slaves } & 49\end{array}$

C Improved protocols of MANA I and their security analysis 50

C.1 Indirect binding and D-H style versions of Improved MANA I . . . . . . . . . . . 50

C.2 Security analysis of the Improved (V-)MANA I protocols . . . . . . . . . . . . . 51

C.2.1 Security analysis of the direct binding improved (V-)MANA I . . . . . . . . . 52

C.2.2 Security analysis of the indirect binding improved (V-)MANA I . . . . . . . . 54

C.2.3 Security analysis of Improved V-MANA I in Diffie-Hellman style . . . . . . . 55 


\section{Introduction}

In this paper, we give a survey of authentication protocols which involve manual transfers of short authentication strings (SASs) over an assumed empirical or authentication channel as might be created by one or more human users of the systems being considered. The careful use of low-bandwidth unspoofable channels offers an interesting alternative solution for the problem of authentication, as opposed to making use of PKI and/or trusted third parties (TTP).

There have been rapid developments in this field in the last few years, resulting in publications, international standards and patent applications relating to a variety of such protocols. In the first few years these protocols were frequently introduced by groups working independently of each other. For example, Stajano and Anderson [47] were the first to attempt to form a secure network for a two-party scenario. The new approach was then studied and refined further by many research authors, most notably Balfanz et al. [3], Creese et al. [9, 10, 11, 12], Gehrmann et al. [13, 14, 15], Hoepman [17, 18], Vaudenay [53], Čagalj et al. [6], Wong and Stajano [56, 57] and Roscoe and Nguyen [32, 33, 37, 41, 42, 43, 44] who introduced both pairwise and group authentication protocols using less human interactions. Creese, Roscoe et al. in $[9,10,11,12,41]$ refer to these human exchanges as empirical channels since the recipient has some empirically based knowledge about the origin of the message, as opposed to cryptographically based knowledge (e.g. via a PKI) of its origin. We therefore use this term throughout this survey.

Given the potential importance of this work, we feel that our survey is timely. ${ }^{1}$ We consider one-way protocols, non-interactive in the sense that all communication is one way, and interactive protocols that work both for pairwise interaction and group formation. Due to the range of potential implementation technologies in this paper we largely abstract away the details that are not immediately important to security. We also have imagined there is a preliminary and insecure group/pairwise set-up protocol (implementation dependent) that is run either before or simultaneously with the first messages of the secure protocol to agree on, for example, the number and identities of protocol participants, since the information will significantly reduce the waiting time in a protocol session.

The development of this novel sort of authentication has arisen from many daily life applications. For example, in the authentication technology, for parties to agree on the same payment records in financial transactions, healthcare information in telemedicine, or cryptographic public keys, their portable devices exchange the data over (insecure) WiFi and then display a short and non-secret digest of the protocol's run that the devices' human owners verbally or visually compare to ensure they agree on the (public) data, i.e. the latter uses human interactions to prevent identity theft. It is thus easier to implement the solutions, because they do not rely on the needs for PIN numbers, passwords or trusted third parties (e.g. the government or security infrastructures distribute ID certificates or private keys to users) which may be too complex and expensive to use on portable devices.

We explain the notation used in describing the protocols as well as a number of cryptographic primitives such as a commitment scheme, short/long-hash functions, and digest functions in Sec-

\footnotetext{
${ }^{1}$ There has been another survey written by Suomalainen et al. [50] where the authors concentrate on pairwise protocols bootstrapping security from scratch by either human interaction or secret shared passwords. As we will see, it is also significantly different from ours in a number of ways: we concentrate on one-way, mutual and group protocols based on human interaction, and classify and analyse them in term of information binding strategies and computational efficiency. In addition, there have been reviews in the papers of Mashatan and Stinson [27], and Pasini and Vaudenay [39, 53], which only look at one-way authentication schemes (both interactive and non-interactive).
} 
tion 2. A simple model for the computation cost of these cryptographic primitives and an attack model are provided to assess the complexity and security of these protocols as we move along.

Although the authentication protocols considered here have been independently introduced by a number of research groups using different notations, this survey will demonstrate that their security is derived from the idea of commitment before knowledge, formally defined in Section 2.2.1. What it does is to force protocol participants to be (jointly) committed to some value before knowing what it is until they reveal their respective shares of the decommitment in a later stage of a protocol run. This committed value will, in turn, always be instrumental in the computation of the $\mathrm{SASs}^{2}$ compared by humans, and therefore the parties' state of knowledge of the SASs is a uniform distribution, i.e. this is the key to defeating any causal influence such as birthday attacks as well as ensuring that search and multiple-shot attacks do not gain any advantage over one-shot and guess attacks. We will see that there are two different approaches of achieving this goal depending on whether the authenticated information is directly or indirectly bound to SASs, as studied in Sections 4.2 and 4.3 respectively. In particular, the direct information binding strategy will be refined by two protocol design principles, termed $\mathbf{P 1}$ and P2, in Sections 4.3 and 5.1.

We start with a number of non-interactive one-way authentication schemes that use empirical channels in different ways, for example: MANA I proposed by Gehrmann, Mitchell and Nyberg [13, $14,15]$. We then see that the scheme neither optimises human effort nor offers as much security as had previously been believed. We offer an improved version that provides more security for half the empirical work, using a more general empirical channel.

In Section 4, we look at a variety of pairwise interactive authentication protocols. We see in order to optimise (i.e. minimise) the amount of human/empirical work done in a protocol, it is better to handle a single SAS rather than the several used by some protocols. Once the human work has been optimised, we turn our attention to minimising the computing power required for the protocols. This is likely to be important in practice because of potential applications in low-power pervasive computing devices.

While there has been much recent literature on pairwise protocols, we find it strange that apart from the authors' group [9, 32, 33, 41] and Valkonen et al. [52] there has been little on group protocols, although there appear to be many potential applications of these. We will discuss group protocols in Section 5 as well as presenting a number of newly invented and modified versions aiming to further improve the processing cost.

In Section 6.1, the computation cost of every protocol will be gathered into three tables that clearly demonstrate two things:

- Interactive schemes (pairwise or group authentication) can be much more efficient in human work than non-interactive ones (one-way authentication);

- Although the security of the majority of protocols rely on the commitment before knowledge idea, the use of direct binding to achieve this goal has a clear advantage in efficiency. This arises from the potential to use a digest function designed to produce only the small number of bits required for empirical comparison as opposed to a conventional cryptographic hash used in indirect binding.

Many of the protocols described in this paper are taken from earlier literature. However we have

\footnotetext{
${ }^{2}$ The committed value could be either used directly as the SAS or inputted as a private key of a digest, universal hash or MAC function.
} 
shown how to make minor improvements to some and major improvements to others. We will use the following notation in protocol descriptions:

- Protocols equivalent to ones from previous literature, though perhaps in different notation, are just cited: [].

- Protocols that have been modified in minor ways, often by replacing one or more cryptographic primitive or other data operation, are cited []*.

- Protocols that are either major modifications to existing ones or just new are marked New.

The protocols in this paper are organised according to their aims (e.g. one-way, pair and group) and structure (direct versus indirect binding). This does not always make it easy to see the way the whole topic has been developed in recent years, frequently by several independent groups. Figure 1 shows all protocols both by year of publication, section number where the protocol is described in this paper and dependence on other work (by citation and directed arrows). For example, an arrow from protocol $A$ to $B$ indicates that the design of protocol $B$ is influenced by $A$.

This paper, particularly as it covers the differing approaches of several research groups and since it sits at the boundary of cryptography and protocol design, requires a lot of (new) notation and definitions. The reader might either choose to study all of this notation in advance, or can read it to the end of Section 2.1 and then refer back to Sections 2.2, 2.3 and 2.4 as needed. ${ }^{3}$

\section{Notation and basic definitions}

Capital letters such as $A, B, C, I$, and $S$ are used to identify parties, and $\forall A$ (or $A^{\prime}$ ) means that a message is sent or received by all parties in a group $\mathbf{G}$ attempting to bootstrap a secure communication between them. In common with much of the literature we are citing, the combination of two pieces of data will frequently be written $x \| y$. This will be synonymous with the ordered pair $(x, y)$.

We will assume each node $A$ in a group $\mathbf{G}$ of $N$ parties has some information $I N F O_{A}$ of length $K$ bits $\left(K / w=K / 32=M\right.$ words $\left.^{4}\right)$ that it wants to have authenticated to other members of the group, this might include:

1. Name and addressing information;

2. Its uncertificated public key or Diffie-Hellman token $g^{x_{A}}$, this might be a long-term object or generated freshly for the present protocol run;

3. Contextual information to help identify it, such as its location or human owner, or the owner's photograph, video and audio;

4. Information (perhaps certificated) relating to its functionality.

\footnotetext{
${ }^{3}$ The reader might go straight to (V-)MANA I protocols and its improved versions in Section 3.2 which give a concrete goal for the best protocol to achieve.

${ }^{4}$ Here we assume that a word consists of $w=32$ bits.
} 


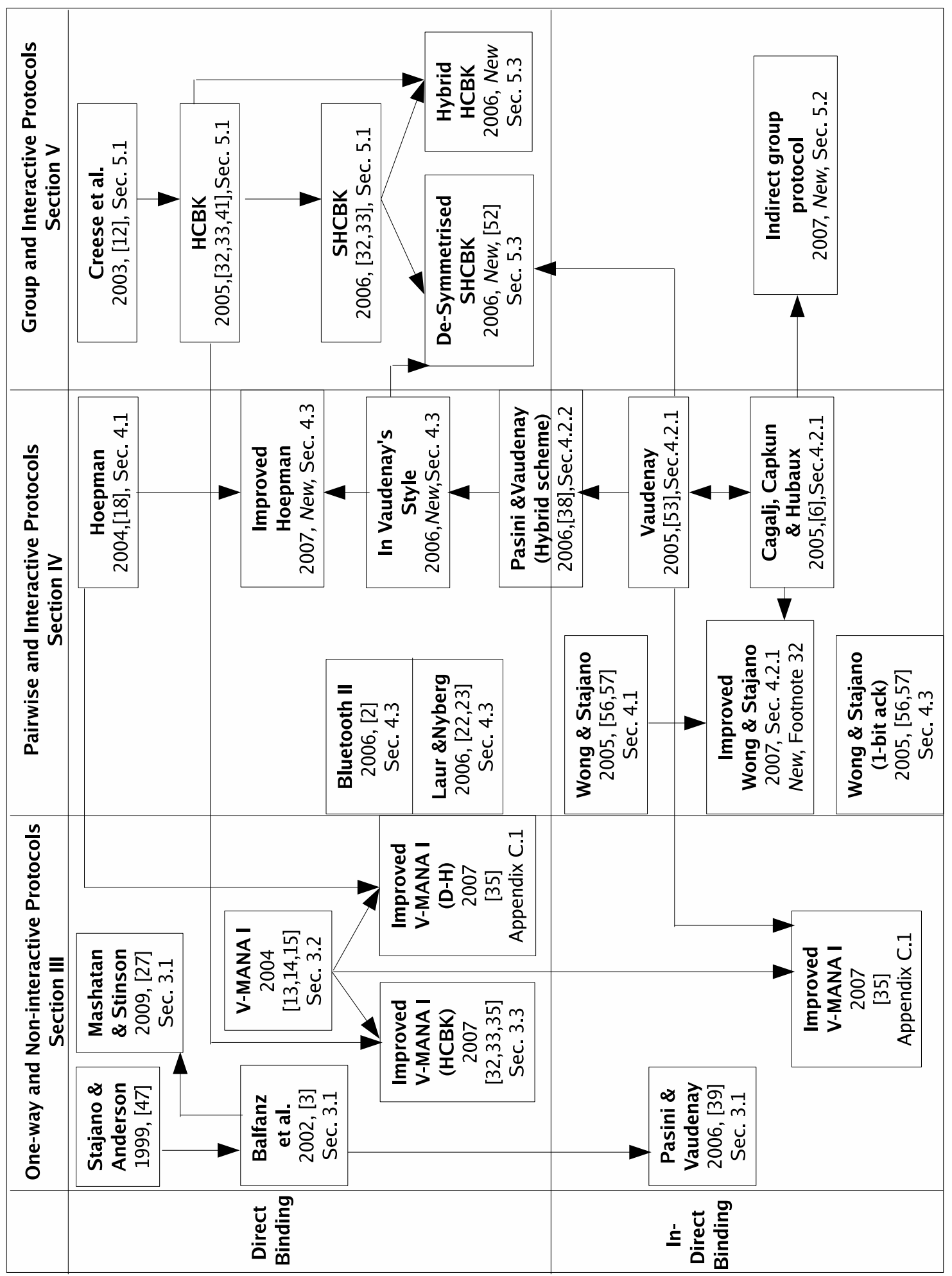

Figure 1: Summary of all protocols. 
Nothing in this information should be secret since all the protocols we consider will make it public. $I N F O_{A}$ might be attached to $A$ permanently or for the long term; alternately some of it might be relevant to this particular run only. The goals of the protocols will always consist of authenticating pairs $\left(A, I N F O_{A}\right)$ as members of the network ${ }^{5}$. In addition, we refer to INFOS as the concatenation in alphabet order of all the distinct pairs parties want to authenticate: $N M$ words if they are all size $M$.

If $I N F O S$ contains $N$ photographs or similar, it may well be of significant size.

\subsection{Communication links}

In some cases, the protocols we quote from other papers are changed in appearance because we seek to use a consistent nomenclature and notation: we do not want a single piece of notation to have inconsistent meanings. One respect in which previous works and papers vary is in the assumptions they make about the empirical or authentic channels. In this paper, we use different notations for communications over a normal Dolev-Yao (insecure) channel and those over four types of empirical ones, which are presented in a descending order of generality.

These empirical channels provide authenticity and data integrity, but not confidentiality: there is enough direct familiarity or physical presence to ensure that the responsibility of a person for a short message can be immediately ascertained.

- $\longrightarrow_{N}$, the normal Dolev-Yao network where all messages transmitted between the laptops in this channel can be overheard, deleted or modified by the intruder. Examples of this channel are the Internet, WiFi, or local network.

- $\longrightarrow W E$, this weak empirical channel cannot be forged, but it can be blocked, overheard, delayed or replayed. This is the weaker of the two forms of empirical channel described in $[39,53]$. A typical example of this channel could be telephone conversation, voice mail or messages, where messages can be delayed, blocked or replayed, but cannot be forged by the intruder.

- $\longrightarrow{ }_{B E}^{t}$, this is similar as $\longrightarrow W E$ except that messages cannot take more than time $t$ to arrive and cannot be replayed. In other words, no empirical message can be accepted more than $t$ time units after it was sent. Such a channel might be implemented over a reliable medium with a known bound on transmission, or over an unreliable one with the addition of some sort of time-stamp. The latter might make sense if the empirical message is sent by some video means, but otherwise would add significantly to the communication burden. We will call this a bounded delay empirical channel.

- $\longrightarrow_{E}$ is the type of empirical channel assumed in $[32,33]$. Messages transmitted over the channel cannot be mistaken or delayed from one to another session. To some extends, this implies that $\longrightarrow_{E}$ is a special case of $\longrightarrow_{B E}^{t}$ where $t$ is chosen to be some lower bound on the length of time between one session and a later one. This is the type we use most often. Sometimes the two-way arrow $\longleftrightarrow_{E}$ is used to indicate (possibly) the same message is transmitted in both directions.

\footnotetext{
${ }^{5}$ We make the identity $A$ explicit here, and in the protocols using it, since the identity is vital to an understanding of who is in the group. In practice, as indicated above, $A$ will normally just be embedded in $I N F O_{A}$. In particular, we assume in these calculations that the name appears in the $K$ bits referred to here.
} 
An example of this channel is manual data transfers [13, 14, 15], i.e. human users manually copy data from one to another device via their in/output interfaces such as screen, monitor and keyboard. In this case, empirical messages cannot be mistaken or delayed from one to another session because: (1) the humans involved are not away at any time during a protocol run, and (2) each device normally only has one in/output interface for displaying or reading data.

- $\longrightarrow S E$, this is similar to a normal empirical channel, but it also provides stall-free transmission. As a result, a message transmitted over the channel cannot be delayed, removed or blocked by the intruder. This implies that $\longrightarrow S E$ is the same as $\longrightarrow_{B E}^{t}$ where $t \approx 0$. We term this a strong empirical channel (or a strong authentication channel). This was also defined in $[39,53]$. Face to face human conversation is an example of this channel.

\subsection{Cryptographic primitives}

We will be using a variety of cryptographic primitives which are related to hashing. Since bitlengths of hash functions vary widely, for clarity, they will be classified relative to three common security properties of a cryptographic hash function, which are inversion-resistance, collision-resistance and second-preimage-resistance. Sometimes they will be subscripted with the number of output bits. For example:

- longhash $(X)$ and $\operatorname{hash}_{B}(X)$ refer to standard cryptographic hash functions [30] which are assumed to be inversion-resistant, collision-resistant and second-preimage resistant. In common with the literature, we will generally assume that they have at least $B=160 \mathrm{bits}$.

- hash $(X)$ and $\operatorname{hash}_{B / 2}(X)$ refer to hash functions which are only assumed to be inversionresistant and second-preimage resistant. Note, $\operatorname{hash}(X)$ is not required to resist collision attacks since its output length is $B / 2$ or around 80 bits.

- $\operatorname{shorthash}(X)$ or $\operatorname{hash}_{b}(X)$ will be functions with sufficiently many bits to offer weak or short-term versions of these security properties of hash $(X)$ or hash ${ }_{B / 2}(X)$. Here $b$ which is the bitlength of shorthash() is in the range of [16,32].

- $\operatorname{digest}(k, X)$ will be a short universal hash or digest of $X$ keyed by $k$ - the specification and purpose of this function will be discussed at length in Section 2.2.2 as well as an additional property often required of the short output hash functions.

- $h_{k}(X)$ will be a universal hash function of $X$ keyed by $k$.

Usually implemented using hashing, we will also use a commitment scheme, whose definition is given below.

\subsubsection{Commitment scheme and Commitment before knowledge}

Definition 1 The following probabilistic commitment scheme, which is adapted from Vaudenay's definition in [53], ${ }^{6}$ consists of two mappings:

\footnotetext{
${ }^{6}$ We note that there is a lack of explicitness in the specification of the commitment scheme defined by Vaudenay in [53], since the security specification there fails to bind it to the input message, as was obviously intended. The
} 
- commit: $\{0,1\}^{K} \times\{0,1\}^{b} \rightarrow\{0,1\}^{B} \times\{0,1\}^{B}$

This mapping takes a public $K$-bit data $I N F O$, a private $b$-bit random nonce $R$ (e.g. $b=16$ ), and then internally generates a $(B-b)$-bit random nonce and produces two $B$-bit strings (e.g. $B=160$ ): a commit value $c$ and a decommit value $d$. This algorithm is nondeterministic due to the internally generated random element of $B-b$ bits.

- open: $\{0,1\}^{K} \times\{0,1\}^{B} \times\{0,1\}^{B} \rightarrow\{0,1\} \times\{0,1\}^{b}$

This mapping takes a public $K$-bit data $I N F O$, a commit value $c$ and a decommit value $d$, and produces an error or success signal together with a $b$-bit random nonce $R$. This algorithm is deterministic and to be such that whenever there exists $R$ such that $(c, d)$ is a possible output for commit $(I N F O, R)$, then open $(I N F O, c, d)$ yields $R$.

The following provides more information about commitment schemes as well as the idea of commitment before knowledge and its restrictive version called joint commitment before knowledge, which underlie the security of nearly every protocol discussed in this thesis.

A commitment scheme used in this paper will have the following two properties:

- $\left(\epsilon_{h}, T_{h}\right)$-hiding: given $(c, I N F O)$, the probability that an attacker can determine the value of $R$ before the decommitment $d$ is revealed is upper bounded by $\epsilon_{h}$ in a time $T_{h}$. When $\epsilon_{h}=2^{-b}$ and $T_{h}=+\infty$ we say that the scheme is perfectly hiding, and this is what we assume in all of the uses of a commitment scheme in this paper.

- $\left(\epsilon_{b}, T_{b}\right)$-binding: the probability that the sender can change the value of INFO to which it has committed once the commitment stage is over is upper bounded by $\epsilon_{b}$ in a time $T_{b}$. When $\epsilon_{b}=2^{-B}$ and $T_{b}=+\infty$ we say that the scheme is perfectly binding, and this is what we assume in all of the uses of a commitment scheme in this paper.

In other words, given $(I N F O, R, c, d)$ where $c \| d=\operatorname{commit}(I N F O, R)$, it must be infeasible to compute a different $I N F O^{\prime}$ such that $c \| d^{\prime}=\operatorname{commit}\left(I N F O^{\prime}, R\right)$, where $d^{\prime}$ can be equal to or different from $d$.

The bitlength of $R$ will be short, e.g. $b \in[16,20]$ or even zero bits, ${ }^{7}$ in all protocols considered. To prevent brute-force search and birthday attacks, the commitment scheme (i.e. commit ()$)$ needs to extend $R$ by a randomly chosen secret nonce $R^{\prime}$ of $(B-b)$ bits so that the combination $R \|$ $R^{\prime}$ has the same bitlength as the output of a cryptographic hash function. This implies that a commitment scheme is designed to be as secure as a standard cryptographic hash function hash ${ }_{160}()$ or longhash(). In practice, a commitment scheme is usually built from a pseudorandom function such as a hash function, and therefore they have the same computational complexity which will be discussed in more detail in Section 2.4.2.

The two above properties regarding security and efficiency of a commitment scheme can be demonstrated by the following construction which was introduced in [40] by Pass.

- Committing: to bind some public information $I N F O$ and a short random secret key $R$ of $b$ bits together, the algorithm picks another secret random nonce $R^{\prime} \in_{\text {random }}\{0,1\}^{B-b}$. It

definition of commit $(\operatorname{INFO}, R)$ there is satisfied by defining the commitment $c=\operatorname{longhash}(N, r)$, where $N$ is a long random nonce internally generated by the committer, and the decommitment $d=(N, r)$.

${ }^{7}$ When random nonce $R$ is zero bit as in the one-way and non-interactive scheme of Pasini-Vaudenay of Section 3.1, we drop $R$ in the notation of a commitment scheme, i.e. commit $($ INFO). 
then sets $d=R \| R^{\prime}$ and $c=\operatorname{longhash}(I N F O \| d)$, i.e. $\operatorname{commit}(I N F O, R)=c \| d$. The committer then publishes the commitment $c .^{8}$

- Decommitting: to decommit or to use open $(I N F O, c, d)=R$, the committer first publishes the decommitment $d$. Anyone can extract $R$ from $d$ (i.e. the first $b$ bits), verify whether $c=$ longhash $(I N F O \| d)$, and then output success or failure.

The conventional understanding of a commitment scheme has been that the committer knows the value to which he or she is committed. In this paper however we will see several cunning uses of the commitment idea but without the knowledge of the committed value from protocol participants, who can be either the committer or other parties. We will see this can be done by distinguishing carefully between when nodes are committed without knowledge to a value and when they know it.

The first use of this idea is called commitment before knowledge, which directly influences the design of HCBK in Section 5.1, and some of the one-way and non-interactive protocols in Section 3.

Commitment before knowledge: ${ }^{9}$ Suppose that, in a partly complete protocol session, a participant $A$ has sent or received parameters, such that in all successful completions of this session some term $d$ has the same value, such as for instance $d=\operatorname{digest}(k, I N F O S)$. Then $A$ is committed to a value of $d$ at this point in the session.

$A$ knows the value of some term $d$ at a point in a partly complete protocol session if, from values $A$ has received, $A$ can compute $d$ without having to invert hash functions or decrypt messages to which $A$ does not hold the decryption key.

$A$ is committed to the value of $d$ before knowing it if the earliest point at which $A$ is committed to a value of $d$ properly precedes the earliest point at which it knows the value of $d$.

The same properties also apply to a more restrictive use of the idea, called joint commitment before knowledge which influences the design of nearly every interactive protocol, i.e. pairwise and group authentication schemes in Sections 4 and 5.

Joint commitment before knowledge: A protocol ensures joint commitment before knowledge for a set of participants if in every sufficiently long partial execution of the protocol, there is a point at which each of those participants is committed to a value for a term $d$, but does not yet know the value of $d$, and moreover in every successful completion of this partial execution, the participants are committed to the same value for $d$.

One simple way to achieve joint commitment before knowledge is as follows: each node is committed to some value by publishing its commitment, for example, by using the commitment scheme described above or a cryptographic hash function. The jointly committed value (i.e. SAS) is then the output of some function, such as summation, exclusive-or, Diffie-Hellman key agreement or digest functions, applying to all of the values to which every node has been committed.

\footnotetext{
${ }^{8}$ Instead of using a cryptographic hash function longhash(), one can switch to universal hash function as defined in Section 2.2, i.e. $c=$ longhash $(I N F O \| d)$ is replaced with $c=h_{d}(I N F O)$. This of course should also apply to decommitting.

${ }^{9}$ We are gratefull to an anonymous referee for wording of this and the idea of joint commitment before knowledge, which improves the versions in earlier drafts of this paper.
} 
We will see later that there are two different strategies of achieving (joint) commitment before knowledge, i.e. direct and indirect information binding approaches which are formally introduced in Section 4. In addition, the combination of commitment before knowledge and direct binding approach will be refined by the principle $\mathbf{P 2}$ to design several group authentication protocols in Section 5.1.

\subsubsection{Short hash and digest functions}

A normal cryptographic hash function is chosen so that it has enough bits to be essentially immune to searching such as the birthday attacks. In this paper, we will see various cunning uses of new functions that, like cryptographic hashes, are intended to randomise and convey no useful information about the preimage. However, their outputs are significantly shorter, since they will always produce values of short authentication strings (SASs) transmitted over the empirical channels which can be very limited in bandwidth.

We will see two variants on this idea: the simpler is what we call a short hash: shorthash(). This has a single argument, and is intended to be uniformly or near-uniformly distributed over its $b$-bit range as its argument varies. Since the hash output is too short, it is impossible to have properties such as collision and inversion resistances like in cryptographic hash functions. What is required instead is the strict avalanche criterion, which states that any number of bit-changes in the input has an equal (but non-negligible) influence on every bit of the output [55].

In some of the protocols we consider, we need to construct a $b$-bit digest of INFOS and some key $k$. Similar to shorthash(), digests cannot have the usually specified properties of a cryptographic hash, namely non-invertability and collision-freeness. On the other hand, we do require that a high degree of randomness arises from the use of the key $k$, as set out below and in $[32,33]$.

Definition 2 [32,33] A b-bit digest function: digest : $K \times M \rightarrow Y$ where $K, M$ and $Y=$ $\left\{0 \ldots\left(2^{b}-1\right)\right\}$ are the set of all keys, input messages and digest outputs, and moreover:

- for every $m \in M$ and $y \in Y, \operatorname{Pr}_{\{k \in K\}}[\operatorname{digest}(k, m)=y]=2^{-b}$

- for every $m, m^{\prime} \in M\left(m \neq m^{\prime}\right)$ and $\theta \in R$ : $\operatorname{Pr}_{\{k \in K\}}\left[\operatorname{digest}(k, m)=\operatorname{digest}\left(k \oplus \theta, m^{\prime}\right)\right] \leq 2^{-b}$

The rationale for these two specifications, especially the use of " $\oplus \theta$ ", will become apparent when we analyse group protocols such as SHCBK in Section 5.1. The digest specification is similar to universal hash functions, except the probability of digest collisions relative to different keys is also considered, i.e. the way digest keys are agreed between nodes in SHCBK protocol can be manipulated such that different nodes' keys may be relatively shifted by a $\theta$ known to the intruder. The inclusion of the $\theta$ shift is therefore to ensure that this type of activity can never benefit the intruder.

Although both shorthash() and digest() are less secure than cryptographic hashes, they are potentially faster to compute, thanks to their short outputs. More details about their comparative speed performance can be found in Section 2.4.2. Designing (universal) hash functions has an exciting and long history in computer science and cryptography, however there does not seems to be much literature on the study and exploitation of short output to speed up computation efficiency. Thus we believe that there is a potential of new constructions for digest functions or adaptation of existing work to acquire computation efficiency, as described below. 
As we will see in the majority of direct binding protocols presented in this paper, the SAS is often the output of a short-output function such as a digest function. For this reason, there have been a number of algorithms proposed to compute short digest $[2,14,15,22,23,33,38]$. To the best of our knowledge, the only ones that explicitly exploit the short output to improve efficiency as well as being proved to satisfy the above specification are based on the idealised framework invented by the authors [32,33], which are adaptations of several well-studied universal hash constructions of Mansour et al. [26], and Krawczyk [20, 21]. We there provide several algorithms using fully random numbers derived from the digest key, which can be simulated in practice by a pseudorandom number generator. The most efficient are based on Toeplitz matrices of bits or words which are generated out of $\epsilon$-biased distribution sequences as pointed out in $[21,33]$. These justify the advantage claimed for short-output digest functions in Section 2.4.2 on the computation cost model. Our algorithms have no restriction on the length of the object (typically INFOS) being digested.

In contrast, several research authors in $[14,15,22,38]$ make use of universal hash functions presented in $[5,19,49]$ to compute digest functions. These algorithms put an upper bound on the input length, and consequently they have to compress a long message into a fixed number of bits (say 512 or 256 bits) by using a cryptographic hash prior to running the algorithms themselves, which turns out to be neither ideally secure nor cost effective [33]. Alternatively, others [2, 38] suggest using first or last $b$ bits of a hash of a large message, which is inefficient and does not necessarily have the precise property we need of being an ideal digest.

\section{$2.3 \quad$ Attack model}

In Section 2, we have defined the intruder's power over data transmitted over all kinds of channels used in the family of protocols. In addition to that, the following are definitions of attacks performed by the intruder, and which will be considered as we move along.

- A general attack: uses (off-line) combinatorial search, e.g. using the birthday paradox to search for hash collisions. This can be either interactive or noninteractive. The attack may consist of multiple protocol runs, and so this is also referred to as multiple-shot or q-shot attack, where $q$ is the number of protocol runs involved.

- A one-shot attack: is a special case of a general attack, i.e. this only involves a single protocol run.

We will also use the term combinatorial search to refer to attacks, whether general or one-shot, which involve combinatorial search, i.e. this is the opposite of a guess attack.

Our aim is to ensure that general or multiple-shot attacks give the intruder no advantage over a one-shot or guess attack on this family of protocols. ${ }^{10}$ This goal is achieved because once every protocol participant is (jointly) committed before knowledge to some short authentication strings (SASs, e.g. a digest value transmitted over empirical channels), then there is not any effective way in which the agents can determine anything about the value - the agents' state of knowledge of the SAS is a uniform distribution.

Moreover, in the majority of protocols considered in this paper, SASs are transmitted over (strong) empirical channels $\left(\longrightarrow_{E}\right.$ and $\longrightarrow_{S E}$ ), and so cannot be mistaken or delayed from one to

\footnotetext{
${ }^{10}$ This is similar to the goal of password-based authentication protocols, which have been studied extensively to date.
} 
another session. ${ }^{11}$ Hence, the SASs in all protocol runs are themselves independent ${ }^{12}$ as pointed out by Vaudenay and other authors $[24,35,39,53]$, and for any $q$ we have:

$$
\operatorname{Pr}(\text { a successful } q \text {-shot attack }) \leq q \times \operatorname{Pr}(\text { a successful one-shot attack })
$$

This model is rather conservative because it is only valid when the intruder launch attacks on many (or perhaps $q$ ) different pairs or groups of parties. ${ }^{13}$ In practice, once a human has noticed a short authentication string disagreement, he or she will be suspicious or aware that an attack is taking place provided implementation is reliably constructed. This will mean that the human will either allow no more attempts or require longer authentication strings, i.e. extending the SAS by 1 bit after each mismatch makes the probability of a successful general attack be upper bounded by $2^{b-1}=\sum_{l=b}^{\infty} 2^{-l}$, where $2^{-l}$ is the likelihood of a successful one-shot attack on an optimal implementation of a $l$-bit SAS protocol, i.e. we will formally define optimality in human interactions of this type of protocols in Section 2.4.1.

Since the protocol design can reduce the probability of a successful attack to the chance of a one-shot attack, for simplicity we will refer successful attacks considered in all protocols to attacks that only involve a single protocol run, i.e. a one-shot attack.

We are also interested in chosen plain-text attacks [46] under which the intruder can influence data trustworthy parties want to authenticate. Although this attack might seem unrealistic, it is desirable that protocols are immune to it, i.e. it will become useful when we analyse protocol of Balfanz et al. in Section 3. Since the attack relies on combinatorial manipulation, we refer to it as a special case of the combinatorial search attack.

In authentication protocols where parties only want to authenticate their public-key-like information, there is no need to distinguish between honest and dishonest nodes. Conversely, every one has to be trustworthy in a key agreement protocol, whether it is pairwise or group schemes. More discussion about this issue could be found at the begin of Section 5 .

\subsection{Cost model}

It seems reasonable to measure the efficiency of the family of protocols in two ways: the amount of empirical or human effort required to complete them; and the amount of processing required at the nodes. The following models for human effort and computation cost are adapted from two of our papers $[32,33]$.

\subsubsection{Human effort}

Our main measure of empirical work is the number of bits of the short authentication strings that are transmitted over the empirical channels. Throughout this paper, we always attempt to optimise the amount of security one can obtain from a given amount of empirical (human) communication. The following definition which specifies when a protocol in this area optimises human interactions

\footnotetext{
${ }^{11}$ Separate security analysis will be provided whenever protocols use weak empirical channels $\longrightarrow W E$ to transmit SASs, which can be stalled and then replayed in other protocol runs.

${ }^{12}$ A bit string $X$ is independent of a bit string $Y$ if for all random variable $X$ of value $x$ and for all random variable $Y$ of value $y: \operatorname{Pr}[X=x]=\operatorname{Pr}[X=x \mid Y=y]$.

${ }^{13}$ We will see later in Appendix C (Theorem 1) how to formalise this statement to give proofs of security for various protocols introduced in this paper.
} 
relative to a level of security obtained has been justified in the papers of the authors [32, 33, 35] and Vaudenay [53].

Optimality of human interactions [32, 33, 35, 53]: A protocol using short authentication strings (SASs) is said to be optimal in human interactions iff there is only a single $b$-bit SAS that needs to be empirically communicated, and the probability of a successful one-shot attack is bounded above by $2^{-b}$.

We will see in later sections that this bound is attainable, provided we can discount the probability of strong cryptographic primitives being broken.

\subsubsection{Computation cost model of cryptographic primitives}

It is essential to optimise the human work in the families of protocols, but at the same time, we also want to minimise the computational cost. We are aware that the cost of agreeing a private key through exponentiation (in Diffie-Hellman's style) or public key cryptography always overtakes the cost of bootstrapping authenticity. However, if the authentication phase is carried out early on lightweight devices prior to key agreement achieved on more powerful devices at a much later stage. Then it is desirable to minimise the computation cost of the authentication protocols done on lightweight devices, whose computation power can be very limited.

In order to assess the complexity of protocols, we have to have a model of the complexity of computing cryptographic primitives, such as a cryptographic hash longhash(), a short hash function shorthash(), a digest function, and a commitment scheme.

Let $B$ and $W$ be the number of bits and respectively words required to hold a long hash value. It is normal that $B=160$ bits, so we assume $W=B / w=5$, here we assume that a word consists of $w=32$ bits. Many researchers in [13,33, 53, 57] suggest 15 or 16 bits are reasonable choices for $b$, the width of the digest output, which is rounded up to 1 word in our analysis. We assume that nonces, keys (used in a commitment scheme and as input of longhash()) and other strong cryptographic values, such as a commitment $c$ and a decommitment $d$, have the same bitlength $B$ and therefore wordlength $W$.

For simplicity, we only look at the Merkle-Damgard construction based hash functions [30] (i.e. block cipher based and customised functions such as SHA-1, MD5, or Davies-Meyer, MatyasMeyer-Oseas and Miyaguchi-Preneel) because they are provably secure given that a one-way and collision resistant compression function exists, and also this family of hash functions is widely used in practice.

It is clear that the cost of computing the $b$-bit output $\operatorname{hash}_{b}(m)$ tends to increase linearly with the length of $m$, since the majority of customised cryptographic hash functions, such as MD5 and SHA-1, are iterative in nature. They are computed by calling a "compression" function once for every (512-bit) block in sequence.

It also seems clear that the cost will increase at least linearly with the output length $b$. Considering the type of operation of the Merkle-Damgard construction reveals that it always has an internal state whose bitlength is equal to or greater than the output bitlength. The internal state is updated by linear or bitwise operators (e.g. Shifting, AND, OR, XOR and rotation) in each loop of the algorithms to ensure that there is a computation between each message input bit and each bit of the internal state, i.e. the strict avalanche criterion. This implies that the computational cost 
is proportional to the size of the internal state, and a simple cost model of a $b$-bit hash function, which we will adopt in this paper, might be:

$$
\operatorname{Cost}\left(h a s h_{b}(m)\right) \approx b \times \operatorname{length}(m)
$$

Since well-known hash algorithms tend to be fixed width and vary significantly in their individual costs, it is hard to be too definite about this rule. ${ }^{14}$ The computational cost model does not take into account the number of clock cycles and implementation-specific (i.e. software or hardware), however it does give an approximate comparison between the cost of computing long and (very) short output functions, for example, cryptographic hash versus digest function as can be illustrated in Table 1.

With respect to the cost of computing a digest. As defined in Section 2.2.2, digest $(\rightarrow,-)$ is a family of short hash functions indexed by a key $k$. Even though the key bitlength might be significantly longer than the hash output in several constructions of universal hash functions invented to date [20, 21], it normally does not play any significant part in the computation. Consequently, key length will not have a big impact on the computation cost. ${ }^{15}$ Hence, we assume the cost model of a digest or universal hash functions is similar to a hash function, which is mainly dependent on the lengths of the input message and the digest output.

A commitment scheme defined in Section 2.2.1 inputs a message of length $K$ bits or $\lceil K / w\rceil=M$ words and a pair of nonces $\left(R, R^{\prime}\right)$ that add up to $B=160$ bits or $W$ words. Since the pair of nonces play the same index role as key $k$ in digest computation, we assume that a commitment scheme takes $M$ words as input. ${ }^{16}$ These are true in both operations used to calculate a commitment and open/verify the commitment. We therefore conclude that the computational costs of computing and verifying a commitment are equal to each other as well as being equal to the cost of computing hash functions. The latter is true because commitment schemes are normally built from pseudorandom functions such as hash functions [40].

Table 1 summarises the computational cost of all cryptographic primitives introduced in this section. While the table might suggest that the cost is equal to this product, what we are actually doing is ignoring the multiplicative constant because all the computational costs come from the same model.

Every protocol in this paper, except the first one in Section 5, only uses long or short hashes, commitment schemes or digest functions. For this reason, we shall apply the simple model to compute the cost for each of them as we move along. In Section 6.1, all of the computational costs and human effort will be put into tables summarising the efficiency of each class of protocols.

\footnotetext{
${ }^{14}$ In practice, one often constructs a variable output-size hash function based on the idea of Key Derivation Function (KDF) or Mask Generation Function. For example, given a 160-bit output hash function such as SHA or MD5, we can use concatenation operator to construct a $160 \times t$-output hash function as follows: $H A S H(m)=h a s h(1, m) \|$ $\operatorname{hash}(2, m)\|\ldots\|$ hash $(t, m)$. This of course clearly follows our computational cost model.

${ }^{15}$ In fact the longer is the key, the fewer the number of random bits we have to generate in our proposed construction of digest functions as well as universal hash constructions of Krawczyk [20, 21], and subsequently the better.

${ }^{16}$ In practice, a commitment scheme, such as one introduced in Section 2.2.1, takes both $M$-word messages and a 160-bit random nonce as its inputs, i.e. these are concatenated before being inputted to a cryptographic hash function: longhash $(I N F O \| R)$. However, it has been noted that longhash $(I N F O \| R)$ could be replaced by a universal hash function keyed by $R$, i.e. $h_{R}(I N F O)$ to reduce the input length, and therefore computational cost. As a result, to give a fair comparative analysis which is independent of implementation, we will stick to this assumption.
} 


\begin{tabular}{|c|c|}
\hline Cryptographic primitive & Computation cost \\
\hline longhash $(I N F O)$ or hash $_{160}($ INFO $)$ & $W M$ \\
\hline longhash $(k)$ or hash ${ }_{160}(k)$ & $W^{2}$ \\
\hline hash(INFO) or $\operatorname{hash}_{80}($ INFO $)$ & $W M / 2$ \\
\hline $\operatorname{shorthash}(I N F O)$ or hash $_{b}(I N F O)$ & $M$ \\
\hline digest $($ INFO $)$ & $M$ \\
\hline $\operatorname{commit}(I N F O, R)$ & $W M$ \\
\hline open $(I N F O, c, d)$ & $W M$ \\
\hline
\end{tabular}

Table 1: In the table, all calculations refer to functions that apply to either a single $I N F O$ of $M$ words or a key $k$ of $W$ words.

\section{Non-interactive protocols}

We examine some protocols attempting to transmit a, possibly very long, message from one party to another efficiently in such a way that the origin and integrity of the message are authenticated. These all use just one-way communication and authentication strings and help to illustrate the power of authenticated empirical channels.

To set this work in context, recall the classic (non-interactive) signature mechanism which works where there is a PKI. Here, a message $I N F O_{A}$ of the sender $A$ is accompanied by the signature $\left\{\text { longhash }\left(I N F O_{A}\right)\right\}_{s k(A)}$. The receiver knows $I N F O_{A}$ really is from $A$, since he can form the cryptographic hash of $I N F O_{A}$ and discover if it really was $A$ who signed this value with her secret key $\operatorname{sk}(A)$. Although the whole of such a message may be assumed to be sent over a standard Dolev-Yao channel, there is in fact a closer tie-in with the subject matter of this section than there might appear to be. For public key encryption and decryption are computationally expensive, there is a strong incentive to keep the bandwidth of information transmitted under this form of cipher to a minimum. We might therefore regard a signature as the combination of a large message $I N F O_{A}$ over an insecure channel with the smaller one longhash $\left(\operatorname{INFO}_{A}\right)$ over an authenticated one.

Since in many cases the empirical channels are human mediated, the chief difference from this view of signature will be that our empirical channels are much lower bandwidth.

\subsection{Long authentication string over the empirical channel}

The above analysis of the use of signatures shows they are closely analogous to the following oneway authentication protocol devised by Balfanz et al. [3]. In this scheme, $A$ wants to authenticate its information $I N F O_{A}$ to $B$.

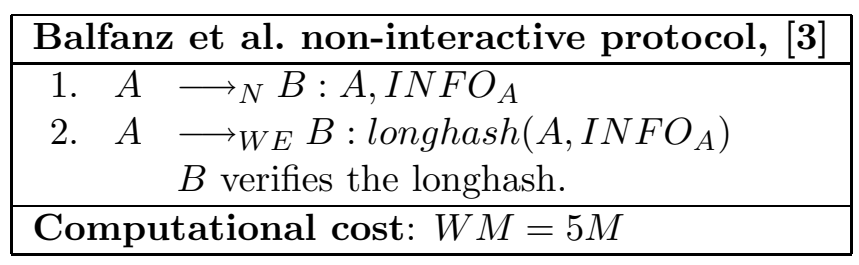

Balfanz et al. [3] did not specify the length of the hash function used. ${ }^{17}$ The main issue we have to

\footnotetext{
${ }^{17}$ In the original protocol [3], there is no restriction on the order of sending and receiving Messages 1 and 2.
} 
decide in analysing this protocol is whether $I N F O_{A}$ might have been manipulated by an attacker. This could be done, for example, by $A$ accepting some piece of externally generated data, such as images and videos, to include in $I N F O_{A}$. In several implementations, such as the specific one anticipated by Balfanz et al. [3] where $I N F O_{A}$ is simply the public key, this attack may be impossible and here an $B / 2=80$-bit hash function suffices. However, we wish to quote a protocol that is secure in general and, following the below analysis taken from $[27,39,53]$, we assume that the hash length is $B=160$ bits. When an intruder can influence some part of $I N F O_{A}$, the intruder can (off-line) search for a different pair $\left(I N F O_{A}, I N F O_{A}^{\prime}\right)$ both yielding the same hash value. $I N F O_{A}$ is then given to $A$ in the information gathering stage, and the intruder sends $I N F O_{A}^{\prime}$ to $B$ masquerading as $A$.

$$
\begin{aligned}
& \text { 1. } A \quad \longrightarrow_{N} I(B): A, I N F O_{A} \\
& I(A) \quad \longrightarrow_{N} B \quad: A, I N F O_{A}^{\prime} \\
& \text { 2. } A \longrightarrow W E B \quad \text { :longhash }\left(A, I N F O_{A}\right)
\end{aligned}
$$

However, this is something which deems infeasible as it must take about $2^{B / 2}=2^{160 / 2}=2^{80}$ computation steps on average to find such a cryptographic hash collision, due to the birthday paradox. What this implies is that even though the protocol is secure, it is not optimal in the human work since $B$ or 160 empirical bits only deliver $2^{B / 2}=2^{80}$ security level.

As a result of a single longhash whose input and output lengths are $M$ and $W$ words, the computation cost is of order $W M=5 M$, thanks to the cost model in Section 2.4.2.

In order to improve the number of authenticated bits, Pasini and Vaudenay [39] make use of a probabilistic commitment scheme ${ }^{18}$ to commit to the authenticated information. The $(B / 2=80)$ bit hash of the commitment is then sent over the weak empirical channel.

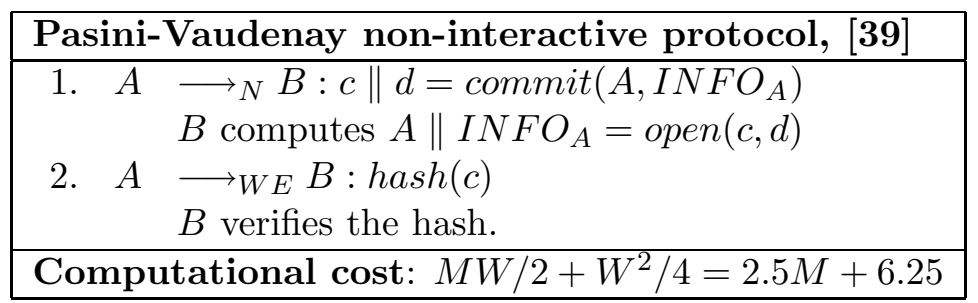

Here the hash function is required to be second-preimage-resistant [30]: an intruder cannot find a second value $v^{\prime}$ such that $h a s h(v)=h a s h\left(v^{\prime}\right)$ for fixed $v$ ) as opposed to collision-resistance required for Balfanz et al. where both $v$ and $v^{\prime}$ are allowed to vary.

In [39], Pasini and Vaudenay argue that this provides the same degree of authentication as the Balfanz et al. protocol, namely $2^{B / 2}=2^{80}$ computation steps, because the probabilistic commitment scheme avoids the possibility of a birthday attack. At the point where $A$ is influenced to use the given $I N F O_{A}$, the intruder cannot know what a nondeterministic component (a hidden random nonce of $B / 280$ bits $=W / 2$ words) that is injected by $A$ into the commitment scheme will be, which is vital in obtaining a collision. Binding the information by a commitment scheme has the advantage of halving the number of empirical bits as well as halving the bitlength of the

\footnotetext{
${ }^{18} \mathrm{~A}$ commitment scheme was defined in Section 2.2.1. We note that there is no random nonce inputted into the commitment scheme used here. Therefore, its bitlength is assumed to be zero, and the commitment scheme has to generate a new long nonce ( $80 \mathrm{bits})$ every time the commit () function is called. This is also the only time when an $(B / 2=80)$-bit commitment scheme is used. For all other employments of a commitment scheme, it is always $(B=160)$-bit.
} 
commitment scheme due to the nondeterminism introduced. These together reduce the cost to $M W / 2+W^{2} / 4=2.5 M+6.25$.

More recently, Mashatan and Stinson [27] introduced another scheme which achieves the same level of security with the same number of empirical bits as Pasini-Vaudenay but does not require the use of a commitment scheme. In the following protocol, $k$ is a long random key of $B / 2=80$ bits which is generated by $A$.

\begin{tabular}{|c|}
\hline Mashatan-Stinson $[\mathbf{2 7}]$ \\
\hline $1 . \quad A \longrightarrow N B: I N F O_{A} \| k$ \\
$2 . \quad A \longrightarrow W E B:$ hash $\left(I N F O_{A} \| k\right)$ \\
\hline Computational cost: $(M+W / 2) W / 2=2.5 M+6.25$ \\
\hline
\end{tabular}

Note, the random key $k$ plays the same role as the non-deterministic component (also of $B / 2=80$ bits) injected by $A$ into the commitment scheme used in Pasini-Vaudenay. This therefore implies that both of these obtain the same level of security. ${ }^{19}$ The computational cost of Mashatan-Stinson is $(M+W / 2) W / 2=2.5 M+6.25$, which is the same as Pasini-Vaudenay. To the best of our knowledge, the above two protocols are currently the best non-interactive schemes in terms of the number of empirical bits relative to the level of security obtained.

The circumstances of non-interactive protocols bring the category of empirical channels $\rightarrow W E$, as opposed to $\rightarrow_{E}$ or $\rightarrow_{S E}$, into question. For unless the recipient knows (s)he is in a protocol and confirms it by some explicit or implicit acknowledgement, how can we possibly state that an empirical message designed for one protocol run cannot be used for a second one? It seems to us that there are in fact three possibilities:

- There is, in fact, no bound on the life of a delayed empirical message. In this case an intruder can block a succession for messages from $A$ to $B$, with the chances of success of each combinatorial search becoming greater as it has more and more empirical messages it can unblock - as in the birthday attack.

It is clear that in any use of delayable empirical channels, one needs to be certain to ensure that this type of storage and re-use cannot occur. The feedback in interactive protocols is one, but in non-interactive channels it is a difficult question: we can avoid re-use through sequence numbers, but if all but one messages are blocked there is no need for re-use for the type of intruder strategy described above to work.

- There might be some mechanism which bounds the life of a delayed message. For example, given sufficiently synchronised clocks, a time-stamp would produce a real bound on the delayability of the message.

Alternately there might actually be some feedback mechanism not explicitly mentioned in the protocol which tells $A$ when her last empirical message has arrived. In the absence of signature mechanism for $B$ that $A$ can trust (unlikely in the circumstances we are considering) this feedback mechanism will probably have to be empirical.

- There may in fact be no significant delay possible: we actually have $\rightarrow S E$. We discuss that case below.

\footnotetext{
${ }^{19}$ Mashatan-Stinson requires that the 80-bit hash function hash() is hybrid-collision resistant as defined in [27].
} 
It seems fair to remark that since even 80 bits will seem tedious for most humans to compare carefully, these one-way non-interactive protocols are not likely to find widespread use. Where it is humans who actually need to do this work: they would need to have a high level of commitment and possibly a well-designed user interface to ensure user compliance. For example, this is particularly a difficult task when 20 hexadecimal-digits (or 80 bits) numbers which need to be compared by humans attempting to set up a secure channels between some wearable sensors and laptops to upload medical data only differ in one or two positions or digits.

\subsection{Short authentication strings over strong empirical channels}

Gehrmann, Mitchell and Nyberg [13] took a different approach to preventing combinatorial search. They use empirical channels to transmit the $b$-bit output of a check function $\mathrm{MAC}_{k}()^{20}$ together with a $b$-bit key that has been instrumental in its computation.

\begin{tabular}{|cc|}
\hline MANA I (Gehrmann, Mitchell and Nyberg), $[\mathbf{1 3}, \mathbf{1 4}, \mathbf{1 5}]$ \\
\hline $1 a . \quad A$ & $\longrightarrow_{N} B: A, I N F O_{A}$ \\
$1 b . \quad B$ & ${ }_{E} A: 1$-bit committed signal \\
2. & $A$ picks a $b$-bit random number $k$ \\
\hline & $\longrightarrow{ }_{E} B: k, M_{k}\left(A \| I N F O_{A}\right)$ \\
\hline
\end{tabular}

To eliminate 1-bit empirical signals in MANA I, ${ }^{21}$ Vaudenay proposes to use a strong empirical channel (stall-free or instant delivery, and is denoted $\longrightarrow S E$ ) to send the key and the check-value. ${ }^{22}$ Thus $2 b$ bits are transmitted in all. This idea turns the protocol into a non-interactive scheme. In the following description, we will modify the scheme slightly by using a digest function to compute the check-value. The rest of this analysis applies to both versions.

\begin{tabular}{|cc|}
\hline V-MANA I, $[\mathbf{5 3}, \mathbf{3 9}]^{*}$ \\
\hline 1. $A$ & $\underset{N}{ } B: A, I N F O_{A}$ \\
& $A$ picks a $b$-bit random number $k$ \\
2. $A$ & $\longrightarrow S E B: k$, digest $\left(k, A \| I N F O_{A}\right)$ \\
& $B$ verifies the digest. \\
\hline Computational cost: $M$ \\
\hline
\end{tabular}

Binding $I N F O_{A}(M$ words) directly to the SAS makes the protocol efficient because each node computes a single digest at a cost of $M$ : much cheaper than a long output hash function in Balfanz et al. $(5 M)$ and a commitment scheme in Pasini-Vaudenay $(2.5 M+6.25) .{ }^{23}$

\footnotetext{
${ }^{20}$ As suggested in $[13,14]$, a check function $\mathrm{MAC}_{k}()$ can be implemented by either CBC-MAC or universal hash functions based on error correcting code, which is potentially less efficient than digest functions as discussed in Section 2.2 .2 and [33]

${ }^{21}$ The 1-bit committed signal, which can be implemented by a red line or a single button, is not a primitive property of empirical channels. It presence aims to indicate to $A$ that $B$ has received Message 1, which could be either original or fake. In the original description of MANA I, the pair of parties additionally need to agree on the success of the protocol with the help of some human interactions. Since this is not important with respect to security analysis, we ignore the step in our description of the protocol.

${ }^{22}$ We can replace the strong empirical channel with a bounded delay empirical one $\left(\longrightarrow_{B E}^{t}\right)$, provided $B$ checks that he has received Message 1 before Message 2 could have been sent.

${ }^{23}$ This measurement only applies to the modified version of V-MANA I, where the digest function is used as opposed to CBC-MAC or longhash functions that will make it increase to $M W=5 M$.
} 
The protocol demonstrates that the use of the strong empirical channel, providing stall-free transmission, will lead to a significant fewer number of empirical bits in non-interactive schemes. Since the uniform distribution property of the digest makes it impossible for the intruder to look for an $I N F O_{I}$ digesting to the same value as $I N F O_{A}$ in ignorance of $k$, this protocol comes close to preventing the intruder from performing any useful combinatorial search.

We note however that the protocol is suboptimal in human work relative to the level of security obtained. Any one can modify $I N F O_{A}$ blindly in the first message and hope that the $b$-bit digests come out the same in the second one. This will occur with a probability of $2^{-b}$ irrespective of the value of the key, which means that $2 b$ empirical bits only guarantee at best a $2^{b}$ security level.

Whilst the security proofs of this protocol given in $[13,15,39]$ are largely correct, what these authors have not discovered is that the bitlength they choose for the key (which happens to be equal to $b$ in this case) is too short compared to the digest output and the authenticated information $I N F O_{A}$. As a consequence, it is impossible to construct a digest, MAC or check-value function such that the probability of any one-shot attack on the protocol is upper bounded by $2^{-b}$. Since the weakness has a very profound impact on all other uses of the digest function, we are going to analyse the (off-line) computation complexity and its related probability of a successful one-shot attack on this protocol. We then deduce a longer key is required in order for the digest function to meet its specification.

We term $b$ and $r$ the bitlengths of the digest output and the key $k$ (in this protocol, $b=r=16$ bits). The intruder first chooses some number $c$ different keys $\left\{k_{1}, \cdots, k_{c}\right\}$. Using an off-line brute force search at the cost of $2^{b c / 2}$ computation steps he can expect to find two different $I N F O_{A}$ and $I N F O_{A}^{\prime},{ }^{24}$ such that:

$$
\forall k \in\left\{k_{1}, \cdots, k_{c}\right\}: \operatorname{digest}\left(k, A \| I N F O_{A}\right)=\operatorname{digest}\left(k, A \| I N F O_{A}^{\prime}\right)
$$

Assuming that the intruder can influence $I N F O_{A}$ that $A$ sends in the first message (i.e. chosen plain-text attacks), there is then an attack it can attempt.

$$
\begin{aligned}
& \text { 1. } A \quad \longrightarrow N \quad I(B): A, I N F O_{A} \\
& I(A) \quad \longrightarrow_{N} \quad B \quad: A, I N F O_{A}^{\prime} \\
& \text { 2. } A \quad \longrightarrow S E \quad B \quad: k \text {, digest }\left(k, A \| I N F O_{A}\right)
\end{aligned}
$$

Recall that in the above protocol, the key length $r$ and digest length $b$ are equal. The following calculations, where these numbers are kept separate, will allow us to draw more general conclusions.

After sending the first message, $A$ picks a random key $k$ : with probability $\frac{c}{2^{r}}, k \in\left\{k_{1}, \cdots, k_{c}\right\}$ and the attack is successful. On the other hand, with probability $\left(2^{r}-c\right) / 2^{r}, k$ is not in this set and the attack is only successful with probability (presumably) $2^{-b}\left(2^{r}-c\right) / 2^{r}$.

Overall, at the cost of $\Theta\left(2^{c b / 2}\right)$ the chance of a successful one-shot attack is:

$$
\mathbf{P r}_{r}(c)=c \times 2^{-r}+\frac{2^{r}-c}{2^{r}} \times 2^{-b}
$$

When $r=b$, this is significantly larger than the desired probability of $2^{-b}$.

\footnotetext{
${ }^{24}$ It might be clearer if we define $H_{\left\{k_{1}, \cdots, k_{c}\right\}}(X)=\operatorname{digest}\left(k_{1}, X\right)\|\cdots\| \operatorname{digest}\left(k_{c}, X\right)$, and if digest is an ideal digest function, then so is the function $H$ with respect to its $c \times b$ output-bits. As there is no limit on the bitlength of the input $X$, it normally takes $2^{c b / 2}$ computation steps to search for a collision, due to the birthday paradox.
} 
The above vulnerability indicates we need to increase the bitlength $r$ of the key to avoid this type of attack. When $r$ increases, $2^{r}$ will quickly become significantly bigger than $2^{b}$ and this will allow the likelihood of a successful one-shot attack $-\mathbf{P r}_{r}(c)$ - to converge to $2^{-b}$. This is however not feasible in this protocol, since the key must be sent with the digest value over the strong empirical channel that is severely limited in bandwidth.

An interesting question arises as we want to know how large the bitlength of the key should be in relation to a fixed amount of information we want to authenticate and the output bitlength of the digest. Since this question is not within the scope of this paper, we point readers to one of our papers [34] where we successfully derive a new combinatorial bound for an almost universal family of hash functions. ${ }^{25}$

This suggests that we should aim always to have key $k$ noticeably longer than the digest in this style of protocol. Of course to do this without ruining efficiency in human effort, we need to find ways of communicating $k$ over a high bandwidth (and insecure) communication link $\longrightarrow_{N}$ rather than empirically.

\subsection{Improved version of (V-)MANA I}

Given two weaknesses discussed in the previous section, we will present improved versions of VMANA I that optimise the use of the expensive strong empirical channel. These improvements can also apply to MANA I. In other words, human comparison/handling of a $b$-bit short authentication string (SAS) always corresponds to probability $2^{-b}$ of a successful one-shot attack. Whilst this can only be done at the expense of introducing another (third) message sent over the Dolev-Yao channel we argue that this is not at all a bad trade-off since our highest priority is to minimise the empirical cost.

In contrast to V-MANA I, the key $k$ generated by $A$ in the following protocol can be as long as we want to ensure that the digest function meets the specification in Section 2.2.2. In addition, we can weaken the assumption that empirical messages' transmission is instantaneous to being of bounded delay as follows.

\begin{tabular}{|cl|}
\hline Improved version of V-MANA I (direct binding) [35] \\
\hline $1 . A \longrightarrow N$ & $B: M$, longhash $(k)$ \\
2. & $A \longrightarrow{ }_{B E}^{t} \quad B:$ digest $(k, M)$ \\
3. $A \longrightarrow_{N} \quad B: k$ \\
\hline Computational cost: $M+W^{2}=M+25$ \\
\hline
\end{tabular}

Note that the message order here, and in other improved schemes of V-MANA I, is more important than in all preceding protocols in this section. We specify that

- To ensure that $B$ was committed without knowledge to key $k$ when Message 2 was sent, $B$ only accepts Message 2 after $t$ time units or more of receiving Message 1.

- To ensure that $B$ was committed to Message 2 when Message 3 was sent, $A$ only sends Message 3 after $t$ time units or more of sending Message 2.

\footnotetext{
${ }^{25}$ There is a known theoretical bound of Stinson [49] on the bitlength of the key that can guarantee the digest meets its specification: $\operatorname{bitlength}(k) \geq \operatorname{bitlength}\left(I N F O_{A}\right)-b$. We should remark that the bound can be met except for an infinitesimal tolerance in the digest collision probability $\epsilon$ for very much smaller lengths than this, see [34]. However, it always has to be significantly longer than $b$ in practice.
} 
Failure to follow these two principles in the implementation of the protocol, each of which uses the time bound on the empirical channel, can result in attacks that involve combinatorial searching.

Interestingly, we can replace the bounded delay empirical channel and the need to wait by a simple acknowledgement from $B$ to $A$. The resulting protocol turns out to be the pairwise (one-way authentication) version of HCBK protocol of Section 5 and [41].

\begin{tabular}{|c|c|}
\hline \multicolumn{2}{|l|}{ Improved version of MANA I (direct binding) } \\
\hline 1a. $A \longrightarrow N \quad B: M$, longhash $(k)$ & \\
\hline 1b. $B \longrightarrow E \quad A:$ 1-bit committed signal & \\
\hline$A \longrightarrow E \quad B: \operatorname{digest}(k, M)$ & \\
\hline 3. $\quad A \longrightarrow N \quad B: k$ & \\
\hline Computational cost: $M+W^{2}=M+25$ & \\
\hline
\end{tabular}

This scheme is flexible since the digest and key (Messages 2 and 3) can be released in any order as long as $A$ has received the commitment signal from $B$ in the first message. It will often be the case that a bounded delay empirical channel and a one-bit acknowledgement signal are alternatives in this style of protocol design/structure.

Since the SAS in these schemes are functionally dependent on the authentic information $M$, we term these as the direct binding version of Improved (V-)MANA I, i.e. direct information binding strategy will be formally defined in Section 4. However, the computation cost is slightly increased to $W^{2}+M=25+M$ due to the extra longhash required in the first message.

Readers who are interested in the formal security proof as well as variants using indirect binding and Diffie-Hellman style can find them in Appendix C or [34].

\section{Interactive protocols}

To authenticate a one-way message, it is obviously convenient to have a non-interactive protocol. We might observe that such a protocol in which the two human participants have to be active at the same time to implement a strong empirical channel (non-delayable) is less attractive: it must be seen as a long way along the road to being interactive. ${ }^{26}$

Interactive protocols, where all parties contribute communications, have two clear advantages of their own. Firstly they can exchange messages without running the protocol multiple times. Secondly, as we shall see, the interaction makes it easier to reduce the number of bits that have to be passed empirically as well as the amount of computation power required at each node.

What we will discover is the significance of the idea of joint commitment before knowledge, introduced by us in Section 2.2.1, in providing the same level of security for all protocols presented in this section, i.e. the probability of a successful one-shot attack ${ }^{27}$ is upper bounded by $2^{-b}$, where $b$ is the bitlength of the SASs. For this reason, in all pairwise schemes (except Hoepman and Wong-Stajano in Section 4.1), the value of the unique SAS is jointly committed to by both protocol participants. This therefore leads us to introduce the two following information binding strategies that help us achieve (joint) commitment before knowledge as well as classifying the many protocols considered in this survey.

\footnotetext{
${ }^{26}$ Perhaps this could be worked around by having a logged recording mechanism for the empirical messages as part of the receiver's system.

${ }^{27}$ More information about the intruder's power and different types of attack can be found in Section 2.3.
} 
- Indirect information binding: A protocol using a SAS is said to achieve indirect information binding if the SAS, jointly committed by every node, is independent of the information INFOS parties want to authenticate.

Typically, to construct indirect binding protocols considered in this paper, the SAS is the exclusive-or of random nonces to which every party has been individually committed at the beginning of each protocol run. In addition, these random nonces are also cryptographically bound to INFOS.

- Direct information binding: A protocol using a SAS is said to achieve direct information binding if the SAS, jointly committed by every node, is dependent on the information INFOS parties want to authenticate.

Typically, to construct direct binding protocols considered in this paper, the SAS is the output of some function applying to INFOS in combination with secret keys individually committed to by every party at the beginning of a run. This binding strategy is also closely related to two protocol design principles $\mathbf{P} \mathbf{1}$ and $\mathbf{P 2}$ introduced later in this survey.

When we study the two strategies in Sections 4.2 and 4.3, we find that direct binding has a clear advantage in efficiency over indirect one. This arises from the potential to use a short output digest function to process the large INFOS as opposed to a conventional long output cryptographic hash functions. The computational efficiency pay-off of direct binding strategy will be illustrated each time a protocol with direct binding is introduced, i.e. we will compare its efficiency explicitly with previous related protocols using indirect binding. The advantage will be demonstrated clearly when the cost of all schemes are gathered in three tables in Section 6.1.

\subsection{Multiple empirical short authentication strings}

We intend to describe two pairwise authentication protocols, the first by Hoepman $[17,18]$ and the second by Wong and Stajano [56, 57], in this subsection. In these schemes, parties manually compare or handle two different short authentication strings (SASs) each of $b=16$ bits, so $2 b=$ 32 bits in all. We point out an important difference in how these two protocols process INFOS.

Hoepman [18] defines SASs as the outputs of a $b$-bit (short) hash function $\operatorname{shorthash}($ ), as mentioned in Section 2.2.2. In addition, the Diffie-Hellman tokens $g^{x_{A / B}}$ play the role of both $I N F O_{A / B}$ and long fresh random nonces, and so must be unpredictable and fresh at each session. 


\begin{tabular}{|c|c|}
\hline \multicolumn{2}{|c|}{ Hoepman pairwise protocol, $[18]$} \\
\hline 1. $A$ & $\longrightarrow_{N} B: \operatorname{longhash}\left(g^{x_{A}}\right)$ \\
\hline $1^{\prime} . \quad B$ & $\longrightarrow_{N} A:$ longhash $\left(g^{x_{B}}\right)$ \\
\hline & Where $x_{Y}$ is a long random nonce of $Y$ \\
\hline 2. $A$ & $\longrightarrow_{E} B: \operatorname{shorthash}\left(g^{x_{A}}\right)$ \\
\hline $2^{\prime} . \quad B$ & $\longrightarrow_{E} A: \operatorname{shorthash}\left(g^{x_{B}}\right)$ \\
\hline 3. $A$ & $\longrightarrow_{N} B: g^{x_{A}}$ \\
\hline $3^{\prime} . \quad B$ & $\longrightarrow_{N} A: g^{x_{B}}$ \\
\hline & $\begin{array}{l}A \text { and } B \text { verify the long and short hashes. } \\
A \text { and } B \text { then share the key } k=g^{x_{A} x_{B}}\end{array}$ \\
\hline 4. $A$ & $\longrightarrow_{N} B:$ longhash $\left(g^{x_{A} x_{B}}\right)$ \\
\hline $4^{\prime} . \quad B$ & $\longrightarrow_{N} A: \operatorname{longhash}\left(g^{x_{B} x_{A}}\right)$ \\
\hline Compr & ational cost: $2(W M+M)=12 M$ \\
\hline
\end{tabular}

This protocol offers a good security, i.e. the probability of a successful one-shot attack is bounded by $2^{-b}$, despite the use of $b$-bit short hashes can be explained through the idea of joint commitment before knowledge: both parties are jointly committed to $g^{x_{A} x_{B}}$ by publishing their shares of the commitment (i.e. longhash $\left(g^{x_{A / B}}\right)$ ) in the first messages. It is therefore vital here that both parties must agree on when to finish inputting the first messages. Once the commitment phase is over, Messages 2, 2' and 3, 3' can be sent out in any order without compromising the security. ${ }^{28}$ We will see an example of what goes wrong without (joint) commitment before knowledge at the start of Section 5.1, which discusses group protocols.

We assume that the word-length of the Diffie-Hellman tokens is $M .{ }^{29}$ Since Messages 4 provide shared secret validation (using longhash() function in this case), they can be neglected in our cost analysis. As a result, each node has to compute 2 longhashes and 2 shorthashes of Diffie-Hellman tokens. Using our cost model of computing hash functions given in Section 2.4.2, the computation cost of Hoepman is of order $2(W M+M)=12 M$, where $W$ and 1 are the output wordlengths of long and respectively short hashes.

Taking a different approach, Ford-Long Wong and Frank Stajano [56, 57] propose another scheme which does not use a short hash function, but does give the same security with an equal number of empirical bits. The simplification comes with an extra cost of more than doubling the input size of the longhash() function used in the commitment phase. This is the consequence of the inclusion of short and long nonces $\left(R_{Y}\right.$ and $\left.K_{Y}\right)$ of $b$ and $(B-b)=(160-b)$ bits, respectively.

\footnotetext{
${ }^{28}$ In [17], Hoepman introduced a modified (pairwise) version of the above scheme in which each party can receive multiple longhashes or commitments from unknown nodes at the very beginning of a run. But (s)he only pairs up with the one, who provides the matched single shorthash shorthash $(X)$ sent over the empirical channel in the second message. In this circumstance, $A$ only sends out the shorthash iff he receives the 1-bit commitment empirical signal from $B$ at the first place and vice versa. Furthermore, these acknowledgement signals must be transmitted over the empirical channel because they must not be blocked or delayed by the intruder. In this version, $A$ does not need to know the identity of $B$ during Messages 1, so Hoepman refers to it as the anonymous case. Whereas the protocol above applies to the non-anonymous case.

${ }^{29}$ As the Diffie-Hellman tokens are the only information parties want to authenticate, we can treat them as $I N F O_{A / B}$ whose lengths are $M$ words. The recommended size of Diffie-Hellman tokens in practice is about 1024 bits which is significantly longer than $W$ words.
} 


\begin{tabular}{|c|c|}
\hline Wong-s & tajano pairwise protocol, $[56]$ \\
\hline 1. $A$ & $\longrightarrow_{N} B: g^{x_{A}}$ \\
\hline $1^{\prime} . \quad B$ & $\longrightarrow_{N} A: g^{x_{B}}$ \\
\hline 2. $A$ & $\longrightarrow_{N} B:$ longhash $\left(A, g^{x_{A}}, g^{x_{B}}, R_{A}, K_{A}\right)$ \\
\hline $2^{\prime} . \quad B$ & $\longrightarrow_{N} A:$ longhash $\left(B, g^{x_{B}}, g^{x_{A}}, R_{B}, K_{B}\right)$ \\
\hline & $R_{Y}$ and $K_{Y}$ are short and long random nonces of $Y$ \\
\hline 3. $A$ & $\longrightarrow_{E} B: R_{A}$ \\
\hline $3^{\prime} . \quad B$ & $\longrightarrow_{E} A: R_{B}$ \\
\hline 4. $A$ & $\longrightarrow_{N} B: K_{A}$ \\
\hline $4^{\prime} . \quad B$ & $\longrightarrow_{N} A: K_{B}$ \\
\hline & $A$ and $B$ verify the longhashes. \\
\hline Compu & tational cost: $2 W(2 M+W)=20 M+50$ \\
\hline
\end{tabular}

The security of this protocol comes from the intruder's inability to invert the longhashes, or to predict the non-determinism introduced by the pair of nonces $\left(R_{X}, K_{X}\right)$ at the point when these are committed to. As in Hoepman, both parties must receive each others' commitments (i.e. longhash) before they reveal their long and short nonces in the third and fourth messages. Once the commitment phase (sending out the longhashes) is over, Messages 3, 3' and 4, 4' can also be transmitted in any order.

With respect to computation cost, while there is no short hash function, the two longhashes (with long inputs) that need to be computed at each node result in a significantly larger cost of $2 W(2 M+W)=20 M+50$ compared to Hoepman $(12 M)$.

We now make two observations about the structure of this protocol. The high cost of computing longhash (due to a long input $\left\langle A, g^{x_{A}}, g^{x_{B}}, R_{A}, K_{A}\right\rangle: 2 M+W$ words) can be improved slightly, as it is sufficient for $A$ to bind $g^{x_{A}}$ to the pair of random nonces $\left(R_{A}, K_{A}\right)$. This leads to the elimination of Messages 1 and 2, and indeed the same problem has been independently found and corrected by the inventors in their revised version of the paper, published in October 2007 [57]. However, they have not noticed that the Diffie-Hellman tokens $\left(g^{x_{A}}\right.$ and $\left.g^{x_{B}}\right)$ can play the dual role of the authentic information and fresh nonces if they are made unpredictable and fresh in each session. For this reason, we can further eliminate the need for long random nonces $K_{A / B}$ to simplify the protocol. A detailed description of our modified version of the protocol will be given in Section 4.2 and Footnote 32.

Both Hoepman and Wong-Stajano are suboptimal in the amount of work required by the humans implementing the empirical channel, since they need to compare more than one string. Whereas the same security level, i.e. the same probability of a successful attack, can be obtained in several ways by them comparing or sending a single SAS of the same length over the empirical channel. This weakness introduces another major disadvantage. If we want to generalise these protocols into multi-party versions then the number of different SASs (each party has to compare or handle manually) would always equal to the total number of nodes: an unattractive prospect for the humans involved!

We end this section with a crucial observation: Hoepman chooses to bind Diffie-Hellman tokens directly to the SASs. This is not the case in Wong-Stajano, which is therefore more expensive in computational cost than Hoepman. By this we mean that the INFOs they are trying to authenticate are used directly in the evaluation of the empirically compared strings in Hoepman, while those compared in Wong-Stajano are not. These two different strategies are termed direct and indirect bindings, and we will explore and compare them in detail when we study protocols 
that can optimise human effort in the sections to come.

\subsection{Indirect binding}

In indirect binding protocols, the SASs, which are jointly committed to and manually communicated by parties, are functionally independent of the information they want to authenticate. This is the idea we have briefly seen in Wong-Stajano, and it appears in many other schemes proposed in the literature $[2,6,22,23,38,53,56,57]$. We will analyse these here. What distinguishes all of these from Wong-Stajano is a single SAS which is required to be compared over the empirical channel as opposed to multiple ones.

While there is no relation between the compared SAS and the authentic information INFO (i.e. they are completely independent in the sense of probability), the security of the protocols comes from some mechanism binding some random nonces, which are instrumental in the computation of SASs, and INFOS together in a secure way. Thus there is a tendency to use commitment schemes (described in Section 2.2.1) in these protocols to obtain that binding.

\subsubsection{Indirect pairwise}

We will discuss protocols covering two different circumstances in bootstrapping security. These were devised by Vaudenay [53] and Čagalj et al. [6] to establish one- and two-way authentication via one- and two-way empirical channels in a peer-to-peer network. We will extend their schemes into group versions in Section 5.2.

The following is the description of a pairwise scheme, invented by Vaudenay [53], that authenticates a single message $I N F O_{A}$ from the party $A$ to $B$ using a one-way weak empirical channel.

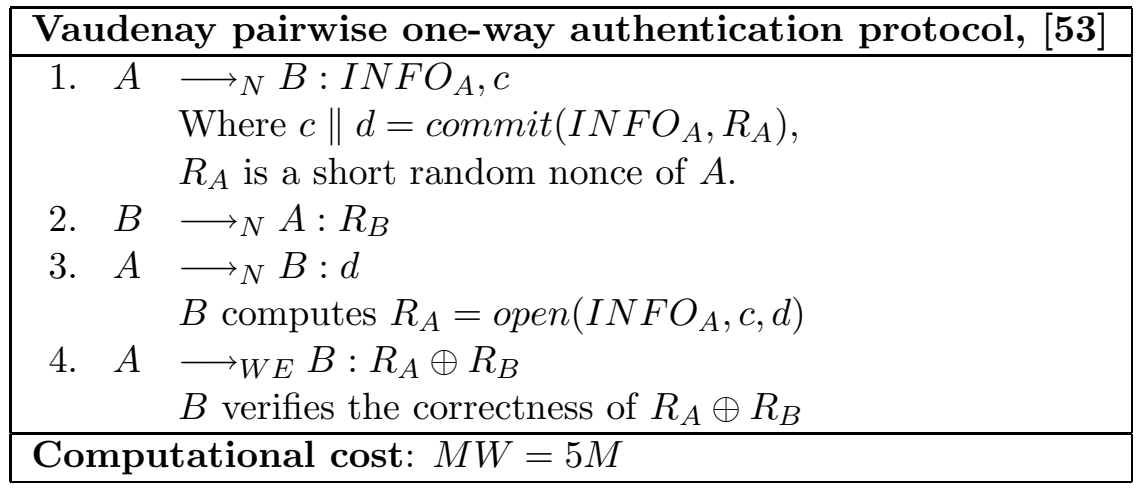

The protocol delivers the guarantee of authenticity of $I N F O_{A}$, and even with a single $b$-bit SAS the probability of a successful one-shot attack is still bounded by $2^{-b}$. This is the consequence of:

- The exchange guarantees the value for $R_{A}$, that $B$ has discovered by using the partial function open(), is the one that $A$ intended.

- The commitment scheme (commit()) has strongly bound the message $I N F O_{A}$ to $R_{A}$ at a point where $R_{A}$ is itself unknown to any attacker.

The above analysis applies to a one-shot attack. If we consider a $q$-shot attack then we need to take into account that the unique SAS of this protocol is transmitted over the weak empirical channel, and so can be stalled and delayed in other protocol runs. With $q$ concurrent runs of $A$ 
and $B$, the number of protocol sessions from the intruder's view will become $q^{2}$. Thus, the chance of a successful $q$-shot attack is upper bounded by $q^{2} / 2^{b}$ as pointed out by Vaudenay [53]. We however argue that the origin of data transmitted over weak empirical channels cannot be forged, and to take advantage of the delayability of SASs the intruder will have to launch attacks on the same pair of parties who are responsible for delivering the SASs, i.e. party $A$ in this case. The above security analysis is therefore only valid with a small value of $q$ because humans are highly sensitive to delays, i.e. they will quickly become aware that an attack is taking place, and so stop any attempt of running the protocol again as pointed out in Section 2.3. In contrast, if we replace $\longrightarrow_{W E}$ with $\longrightarrow_{E}$ then a SAS cannot be delayed from one to later runs, and so to have a fair chance of a successful attack, the intruder needs to run $2^{b}$ concurrent runs of (perhaps different) pair of parties: an $2^{b}$-shot attack.

There is a single commitment used (committed by $A$, and decommitted or opened by $B$ ), hence the computing cost at each node is of order $M W=5 M$. Here, both a commitment $c$ and a decommitment $d$ have the same length of $W$ words. ${ }^{30}$

Although Vaudenay's scheme halves the amount empirical communication relative to Hoepman and Wong-Stajano, it only provides one-way authentication representing one role of pairwise schemes in this paper. In practice, we often want to achieve more than this, and that is why we now consider another protocol performing message authentication in both directions at the same time. Suppose $B$ has some $I N F O_{B}$ and wants to have it authenticated to $A$, then the natural way to tackle this problem is to make $B$ commit to its information as done by party $A$. This idea, proposed by Vaudenay in Appendix A of [53], fortunately makes the protocol structure completely symmetrical. It is essentially the same as another protocol which is termed DH-SC and invented by Čagalj, Čapkun and Hubaux [6].

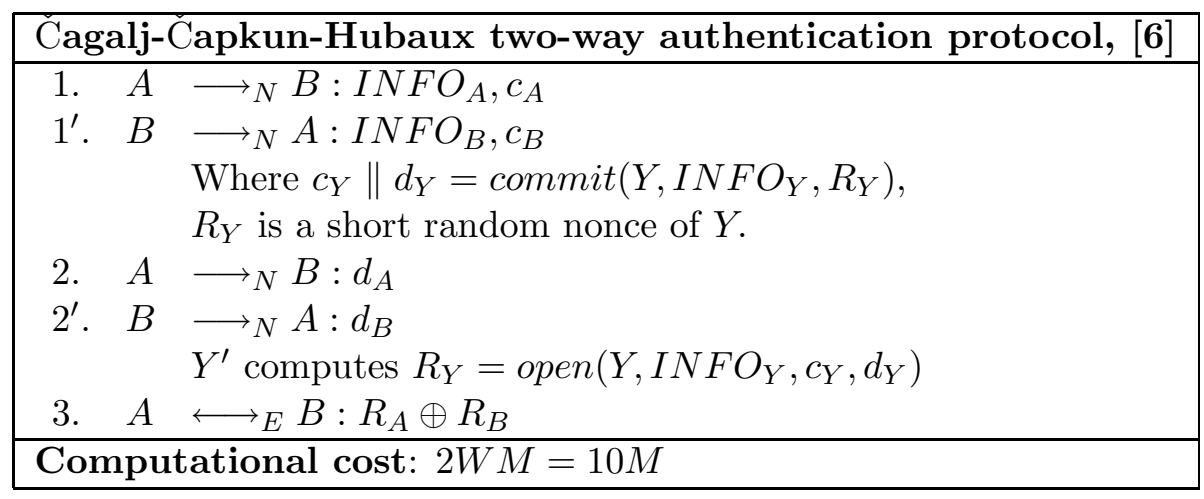

Both of the above protocols use the joint commitment before knowledge principle to precommit two parties to the XOR of some random short secrets or nonces. This is achieved by parties outputting their shares of the commitment to each other in the first messages.

This scheme can be regarded as an upgraded version of Wong-Stajano (Section 4.1) in two ways. Firstly, the two initial messages in Wong-Stajano have been successfully eliminated. This is based on the ground that each node $A$ only needs to commit to its $I N F O_{A}$ at the beginning, so he has not to acquire $I N F O_{B}$ at the time of computing the commitment. Secondly, the order of releasing the SAS and the decommitments has been reversed relative to Wong-Stajano. ${ }^{31}$ As a consequence,

\footnotetext{
${ }^{30}$ The cost of XORing two short random nonces $R_{A}$ and $R_{B}$ is small compared to implementing the commitment scheme, and therefore is neglected here.

${ }^{31}$ The SAS and the decommitments here correspond to the two different short nonces and the long nonces in
} 
parties only need to manually compare a single SAS, which is the XOR of short nonces $R_{A}$ and $R_{B}$ implicitly derived from the decommitments.

It is interesting to note that the same technique can be used to improve the human and processing cost of Wong-Stajano. ${ }^{32}$

Regarding computation cost, the two commitments would double the cost of Vaudenay to an order of $2 W M=10 M$. On the other hand, if we quantify the cost relative to the amount of information authenticated then Vaudenay and Čagalj-Čapkun-Hubaux will be equal to each other. The result illustrates the gain in efficiency of these in comparison with Hoepman and Wong-Stajano in Section 4.1.

Another advantage of Čagalj-Čapkun-Hubaux is that the symmetrical structure and a single SAS subsequently led us to realise the possibility of generalising it into a group version, as described in Section 5.2.

\subsubsection{Hybrid protocol}

Prior to discussing direct binding protocols, we describe an important scheme bridging the gap between the two strategies both in terms of protocol structure and computational cost. Pasini and Vaudenay [38] propose a two-way authentication protocol using the idea of Vaudenay's one-way scheme in Section 4.2.1. They make use of a truncated hash function that we have improved to a digest and a symmetric empirical channel.

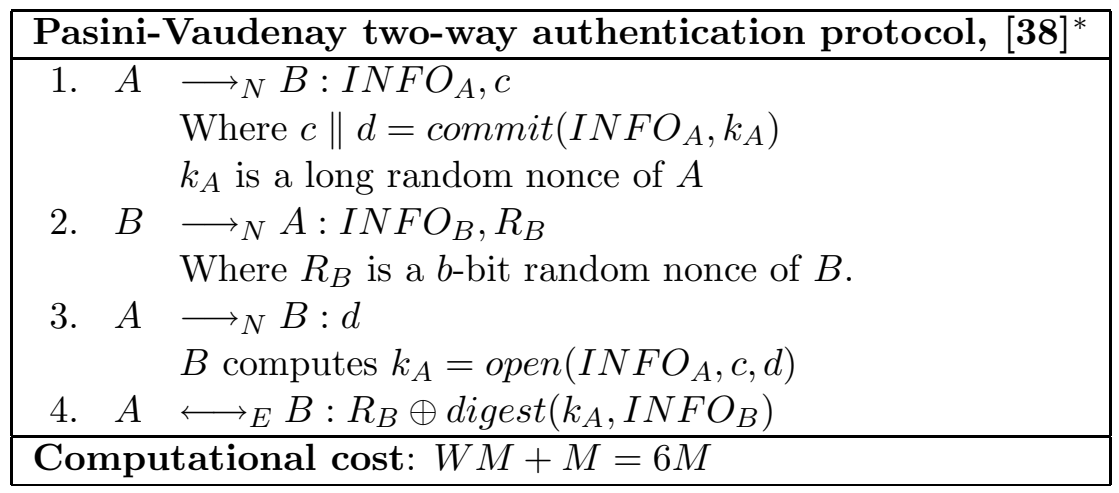

Though the SAS $=R_{B} \oplus \operatorname{digest}\left(k_{A}, I N F O_{B}\right)$ depends functionally on $I N F O_{B}$, it is probabilistically independent of $I N F O_{A}$. This observation makes the scheme stand as a hybrid of direct- and

Wong-Stajano, respectively.

${ }^{32}$ The idea of eliminating the first two messages carrying $g^{x_{A} / B}$, removing the long random nonces as well as reducing the number of different SASs to a single one of $b$ bits in Wong-Stajano can be demonstrated by our revised scheme. The scheme achieves the same level of security as Wong-Stajano in Section 4.1, i.e. the probability of a successful one-shot attack is bounded by $2^{-b}$.

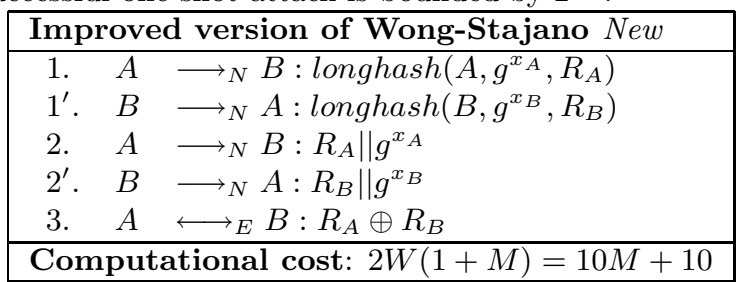

Since there are two longhashes each node has to compute, the computation cost of this scheme is $2 W(1+M)=$ $10 M+10$ which is less than a half of the original Wong-Stajano protocol $(20 M+50)$. 
indirect-binding protocols. Interestingly, the hybrid strategy is also reflected by the differences in the bitlengths and the functionality of the two random nonces: $R_{B}$ and $k_{A}$. $R_{B}$ is protected by the structure of the protocol from guessing attacks and so can be short, whereas $k_{A}$ is not and so has to be long; the two influence the final empirical string in different ways.

There is no need to use a commitment scheme to bind $I N F O_{B}$ to $R_{B}$, so each node needs to compute a digest and either a commitment or a decommitment. The processing cost drops to $W M+M=6 M$, thanks to the efficiency of a digest function, ${ }^{33}$ which is significantly cheaper than Čagalj-Čapkun-Hubaux $(10 M)$ which is the fully indirect binding scheme.

\subsection{Direct binding pairwise protocols}

The direct binding approach requires the SAS, to which every party is jointly committed without knowledge, to be dependent on the information they want to authenticate. The Hoepman protocol that we have already studied falls into this category, but is not optimal in the human work. In this section and later ones we will discuss a number of other pairwise and respectively group protocols which are optimal in this respect. It should be noted, however, that any group protocol can be used to create a group of size 2 , and, as we shall see, do so as efficiently as the ones in the present section.

Direct binding has been shown in two different situations to have an advantage in computation cost over indirect: Hoepman (direct) versus Wong-Stajano, and Pasini-Vaudenay (half direct or hybrid) versus Čagalj-Čapkun-Hubaux.

Our first task is to formalise the direct binding approach as the following principle $\mathbf{P} \mathbf{1}$, introduced by us in $[32,33]$.

P1 $[32,33]$ All the parties intended to be part of a protocol run should agree over an empirical channel on a short-output hash or digest of all the information that the parties want to authenticate. This method maximises the security of the authentication for a given amount of work on the empirical channel, and it leads to protocols that are as efficient computationally as any alternatives, and frequently considerably more efficient.

In all the protocols we introduce in this paper, the "complete description of the run" is identified with INFOS, the collection of all the information that any member of the group wishes to have authenticated to it: the concatenation of pairs of the form $\left(A, I N F O_{A}\right)$. Once the agreement required in $\mathbf{P} \mathbf{1}$ has occurred, unless there is a hash or digest anomaly - different nodes in the group computing the same hash value or digest from different antecedents - clearly all the parties agree on all the data transmitted during the protocol.

In this section, a number of protocols providing mutual authentication are presented in an ascending order of computation efficiency and simplicity. In addition to the common use of the direct binding strategy to obtain joint commitment before knowledge, they are all asymmetrical in structure, which is similar to the one-way authentication protocol of Vaudenay [53] in Section 4.2.1.

Unlike indirect binding schemes, parties need to generate fresh long random nonces or sub-keys, which have enough entropy to prevent them from being subject to a combinatorial search. ${ }^{34}$ On the other hand, the security analysis of the direct binding schemes is similar to indirect binding

\footnotetext{
${ }^{33}$ There should have been no improvement $(W M+W M=10 M)$, if we had not switched to the use of digest.

${ }^{34}$ It is possible to regard these long fresh sub-keys as the extended versions of short random nonces, used in commitment schemes in indirect binding protocols.
} 
ones provided the digest function is ideal, as specified in Section 2.2.2. In every case, the protocols have the same security (i.e. the probability of a successful one-shot attack is bounded by $2^{-b}$ ) because nodes (and hence the intruder) do not know the final value of the digest key $k$ until they are committed to the final value of the digest, truncated hash or universal hash output, thanks to the joint commitment before knowledge idea that provides a common theme to this family of protocols.

Since the roles of the sub-keys and INFOS are different in digest computation, we will analyse how sub-keys are combined into a single digest key as we move along.

The following protocol is taken from Bluetooth whitepaper [2], where $k_{A}$ and $k_{B}$ are long fresh sub-keys generated by $A$ and $B$.

\begin{tabular}{|ccc|}
\hline \multicolumn{3}{|c|}{ Bluetooth 2, [2] } \\
\hline 1. & $A$ & ${ }_{N} B: I N F O_{A}$ \\
$1^{\prime}$. & $B$ & ${ }_{N} A: I N F O_{B}$ \\
2. & $B$ & ${ }_{N} A:$ longhash $\left(\right.$ INFOS,$\left.k_{B}\right)$ \\
3. & $A$ & ${ }_{N} B: k_{A}$ \\
$3^{\prime}$. & $B$ & ${ }_{N} A: k_{B}$ \\
& & $k_{Y}$ is a long fresh key of $Y$ \\
4. & $A$ & ${ }_{E} B: \operatorname{trunc} c_{b}\left(\right.$ longhash $\left.\left(f\left(k_{A}, k_{B}, I N F O S\right)\right)\right)$ \\
\hline Computational cost: $W(2 M+W)+2 W(M+W)=20 M+75$ \\
\hline
\end{tabular}

In this protocol, the sub-keys of $A$ and $B$ are concatenated with INFOS: $f\left(k_{A}, k_{B}, I N F O S\right)=$ $k_{A}\left\|k_{B}\right\| I N F O S$. Since the operator is not commutative, parties have to arrange the sub-keys in the same order in which the distinct $\left(A, I N F O_{A}\right)$ s are concatenated into a single INFOS.

The inefficiency in using a truncated hash function ${ }^{35}$ will increase the computation cost of the above "Bluetooth 2" to $W(2 M+W)+2 W(M+W)=20 M+75$ as opposed to $W(2 M+W)+2 M=$ $12 M+25$ should we employ a digest and XOR to combine sub-keys. Unfortunately, the latter will still be more expensive than the related protocols using indirect binding (Čagalj-Čapkun-Hubaux: $10 M$, and Pasini-Vaudenay: $6 M$ ) and the two following schemes, as it is redundant to bind INFOS and sub-keys together by using both longhash function in Message 2 and in the SAS. Either of them would be sufficient for the obtained security.

Removing this unnecessary binding in Message 2 of Bluetooth 2 can increase its computational efficiency as well as simplicity (eliminating Messages 1 and $1^{\prime}$ ), since $B$ does not need to know $I N F O_{A}$ (and INFOS) at the point when he is committed to $k_{B}$. This is what was proposed in $[22,23]$ by Laur and Nyberg:

\begin{tabular}{|c|c|}
\hline \multicolumn{2}{|c|}{ Laur-Nyberg pairwise protocol, $[22,23]$} \\
\hline \multicolumn{2}{|c|}{ 1. $A \longrightarrow_{N} B: I N F O_{A}, c$} \\
\hline & Where $c \| d=\operatorname{commit}\left(k_{A}\right)$ \\
\hline 2. $B$ & $\longrightarrow_{N} A: I N F O_{B}, k_{B}$ \\
\hline 3. $A$ & $\longrightarrow{ }_{N} B: d$ \\
\hline & $B$ computes $k_{A}=\operatorname{open}(c, d)$ \\
\hline 4. $A$ & $\longleftrightarrow_{E} B: h_{k^{*}}(I N F O S)$ \\
\hline & Here $k^{*}=g\left(k_{A}, k_{B}\right)$ \\
\hline Coml & tational cost: $2 W M=10 M$ \\
\hline
\end{tabular}

${ }^{35} \operatorname{trunc}_{b}()$ takes the first $b$ bits of its input. 
Here $h_{k^{*}}()$ is a universal hash function [49] of the appropriate length, i.e. $b$-bit in this case, whose specification is closely related to a digest as discussed in Section 2.2.2. The impact of removing redundancy can be seen in the decline of the computation cost of this protocol: $W^{2}+2 W M=$ $25+10 M,{ }^{36}$ which can be improved further because Laur-Nyberg has not exploited the short bitlength of SAS to increase efficiency.

Unlike Bluetooth 2, Laur and Nyberg use a different function $k^{*}=g\left(k_{A}, k_{B}\right)=\left(k_{A}^{1} \cdot k_{B}\right) \oplus k_{A}^{2}$, using (polynomial) multiplication over a finite field $\mathrm{GF}\left(2^{r / 2}\right)$ to combine sub-keys. Here $k_{A}^{1}$ and $k_{A}^{2}$ are the first and second halves of $k_{A}$. Not only is this method expensive with long keys compared to concatenation and exclusive-or as we are going to propose, but also the parties need to agree an irreducible polynomial of order $r / 2$ prior to each session.

We observe that it would be equally satisfactory to use the combination of $k_{A} \oplus k_{B}$ and a digest function in place of $h_{k^{*}}(I N F O S)$, resulting in an improvement of computational cost $\left(W^{2}+2 M=\right.$ $25+2 M)$ which is approximately 5 times cheaper than Cagalj-Čapkun-Hubaux $(10 M$, the related protocol using indirect binding) should $M$ gets large. This clearly demonstrates the advantage in efficiency of direct binding protocols over indirect binding ones.

After this transformation and a replacement of a commit scheme with a longhash, the protocol becomes similar to the following, which also has the same cost of order $25+2 M$. This was discovered independently by the author in the summer of 2006 when we combined ideas used in our SHCBK protocol (see Section 5.1) and Vaudenay's protocols (see Section 4.2.1).

\begin{tabular}{|l|}
\hline Pairwise authentication scheme in Vaudenay's style New \\
\hline $1 . A \longrightarrow_{N} B: I N F O_{A}$, longhash $\left(k_{A}\right)$ \\
$2 . B \longrightarrow_{N} A: I N F O_{B}, k_{B}$ \\
$3 . A \longrightarrow_{N} B: k_{A}$ \\
$4 . A \longleftrightarrow_{E} B: \operatorname{digest}\left(k_{A} \oplus k_{B}, I N F O S\right)$ \\
\hline Computational cost: $W^{2}+2 M=2 M+25$ \\
\hline
\end{tabular}

We subsequently discovered that the same ideas could be used to devise a more efficient version of the Hoepman protocol, which halves (and optimises) the amount of human work while achieving the same level of security, i.e. the probability of a successful one-shot attack is bounded by $2^{-b}$ :

\begin{tabular}{|l|}
\hline Improved Hoepman New \\
\hline $1 . A \longrightarrow_{N} B:$ longhash $\left(A, g^{x_{A}}\right)$ \\
$2 . B \longrightarrow_{N} A: g^{x_{B}}$ \\
$3 . A \longrightarrow_{N} B: g^{x_{A}}$ \\
$4 . A \longleftrightarrow_{E} B:$ shorthash $\left(g^{x_{A}} \oplus g^{x_{B}}\right)$ \\
\hline Computational cost: $W M+M=6 M$ \\
\hline
\end{tabular}

The main difference between this and the previous schemes is that there is no $I N F O_{A / B}$ because the Diffie-Hellman tokens play the dual-role of both $I N F O_{A / B}$ and the long secret keys. In order for the protocol to be secure, the Diffie-Hellman tokens must be fresh at each session and unpredictable. ${ }^{37}$ Also because of this, the digest function (2-input function) can be replaced by a single input short hash function shorthash(); though the combination of this and the exponentiation of Diffie-Hellman

\footnotetext{
${ }^{36}$ We choose to ignore the cost of computing $g()$ to combine sub-keys in this calculation, since it is negligible relative to the computation of a universal hash, which involves applying a cryptographic hash to the $2 M$-word input message $I N F O S$ in the first place, as specified by Laur and Nyberg in $[22,23]$, which results in a cost of $2 W M=10 M$.

${ }^{37}$ It is possible but not necessary to replace $g^{x_{A}} \oplus g^{x_{B}}$ with $g^{x_{A} x_{B}}$ in this scheme.
} 
needs to satisfy a specification similar to that of the digest function and the randomising effect of XOR in combining $k_{A}$ 's.

In comparison with Hoepman, this requires a single SAS halving the human work. As in previous protocols, the computation of one longhash and one short hash of Diffie-Hellman tokens results in a cost of $W M+M=6 M$ : exactly a half of Hoepman (direct binding, (12M) and significantly lower than the improved version of Wong-Stajano (the related protocol using indirect binding, $10 M+10$ ) of Footnote 32.

It is worth thinking for a moment about how a two-way agreement of a short string or similar occurs between a pair of people over an empirical channel. There are likely to be few situations where the string needs to be communicated both ways: all that is necessary is for one party to communicate it to the other, who checks equality with the data displayed on her machine and then tells the first of the agreement, i.e. sending a 1-bit committed signal over the empirical channel. We might therefore structure the above protocol as follows.

\begin{tabular}{|l|}
\hline $\begin{array}{l}\text { Improved Hoepman' } \\
\text { (One-way empirical channels) }\end{array}$ \\
\hline $1 . A \longrightarrow_{N} B:$ longhash $\left(A, g^{x_{A}}\right)$ \\
$2 . B \longrightarrow_{N} A: g^{x_{B}}$ \\
$3 . A \longrightarrow_{N} B: g^{x_{A}}$ \\
$4 . A \longrightarrow_{E} B:$ shorthash $\left(g^{x_{A}} \oplus g^{x_{B}}\right)$ \\
$5 . B \longrightarrow \longrightarrow_{E} A:$-bit committed signal \\
\hline Computational cost: $W M+M=6 M$ \\
\hline
\end{tabular}

And we could re-structure just about all the protocols in this paper similarly.

Once $A$ has received Message 2 from $B$, he can send Messages 3 and 4 in any order.

Wong and Stajano [57] give a protocol using this separated structure explicitly; it is however more expensive at $W(2 M+1)=10 M+5$ as well as requiring another 1-bit empirical signal in Message 3:

\begin{tabular}{|c|c|}
\hline \multicolumn{2}{|c|}{ Wong-Stajano (One-way empirical channel), [57] } \\
\hline 1.A & $\longrightarrow_{N} B: g^{x_{A}}$ \\
\hline $2 . B$ & $\longrightarrow_{N} A: B, g^{x_{B}}, M A C_{K_{B}}\left(B, g^{x_{A}}, g^{x_{B}}, R_{B}\right)$ \\
\hline & $R_{B}$ and $K_{B}$ are short and long random nonces of $B$ \\
\hline 3. $A$ & $\longrightarrow_{E} B:$ 1-bit committed signal \\
\hline 4. $B$ & $\longrightarrow{ }_{E} A: R_{B}$ \\
\hline $5 . B$ & $\longrightarrow_{N} A: K_{B}$ \\
\hline 6. $A$ & $\longrightarrow_{E} B: 1$-bit committed signal \\
\hline
\end{tabular}

The order of Messages 4 and 5 can be interchanged in this protocol. The pair of Messages 4 and 6 results in symmetric agreement on $R_{B}$ : in fact they are just an implementation of " $A \longleftrightarrow_{E} B$ : $R_{B} "$.

It seems unlikely that the computation cost of the cheapest of these protocols can be reduced much further. It also seems clear that some sort of cryptographic binding of INFOS to the empirical message is necessary, and our assumed model of the digest function appears to be a lower bound on that as we want to bind the whole of INFOS. Similarly, it is clear that for the joint commitment before knowledge approach to work, we need to have a token randomising the SAS value and being committed to before any node knows it. This has to be done with strong cryptography, and the hash used in, for example, Laur-Nyberg appears to be as efficient as possible 
at doing this.

Another observation we want to make is that in the above protocols the use of a strong cryptographic primitive, such as a hash function or a commitment scheme, to protect the secrecy of long random nonces or keys, and in the mean time a much shorter (and therefore weaker) function to digest large INFOS clearly aims to block combinatorial search and guess attacks separately. This idea is called separation of security concerns which has been discussed in detail by us in [35].

\section{$5 \quad$ Group protocols}

The majority of work done in bootstrapping security in pervasive computing to date has focused on pairwise applications in a peer-to-peer network. However, we believe there is a similar potential for bootstrapping security in larger groups as can be shown by the following example. A group of people, who are present in the same location, might want to transfer data between them securely, meaning that they want it to be secret and of authenticated origin. They all have some pieces of computing hardware (e.g. a mobile phone or a PDA). However, none of them knows the unique name of any of the others' equipment, and in any case there is no PKI which encompasses them all.

Work in this area seems so far to have been restricted to the author's group (including Roscoe, Creese, Goldsmith and Zakiuddin), and more recently Valkonen et al. This has resulted in several group protocols presented in $[9,10,11,12,32,33,41,52]$. In $[32,33]$, we identified the main challenges of bootstrapping group security in pervasive computing, and these can be explained as follows.

There is a slightly grey area for protocols building groups of more than 2. Should we or should we not be content if the presence of a corrupt party in a group means that communications between other trustworthy members of the group are themselves compromised? In some of the circumstances, where we may wish to use ad hoc group formation protocols, it would be much better if the protocols were tolerant of corrupt members. We will, therefore, be careful about our assumptions on this front. It is obvious any key agreement protocol is at least partially compromised by the presence of a corrupt participant. However, protocols which merely authenticate public-keylike information are not automatically compromised: they could be said to be establishing a local PKI. As we will see, this will be successfully resolved by using the idea of (joint) commitment before knowledge.

The issue of scalability plays a crucial role in constructing group protocols because of the limited computation power of lightweight devices, and the fact that the amount of human work required will inevitably grow as the size of the group does. Our priority is still to optimise the human work relative to the security obtained. The best we can hope for is the same as in the binary case: it might be possible for a group to manually compare a single short authentication string (SAS) of $b$ bits, and obtain the same $2^{b}$ level of security.

We have already seen that the direct information binding strategy is significantly more efficient than indirect one for pairwise protocols (i.e. both of these information binding strategies are instrumental to achieving commitment before knowledge as pointed out in Section 4). In this section, we will see the same is true for group protocols, namely HCBK and SHCBK versus the group version of the indirect binding pairwise protocol of Čagalj-Čapkun-Hubaux. Interestingly, it is possible to further improve the efficiency in direct group protocols with a trade-off between 
human and computational costs, or by making use of protocol structure of the one-way scheme of Vaudenay [53].

\subsection{Some existing direct binding group protocols}

The following protocol introduced by Creese et al. [11] is probably the very first group authentication protocol in the area of pervasive computing. Here, $\forall A$ means that a message is sent to, or received by, all parties in the group $\mathbf{G}$ attempting to achieve a secure link between their laptops or PDAs. $P k_{A}$ stands for an uncertificated public key that $A$ wants to authenticate to the group, whereas $T_{A}$ and $N_{A}$ are $A$ 's fresh nonces. The superscript 'all Messages $2^{d}$ ' represents the concatenation of all the decrypted content of Messages 2 in alphabetical order, for example. In addition, Messages 4 do not add any extra security to the scheme, its presence aims to provide a confirmation of the shared secret information. ${ }^{38}$

\begin{tabular}{|lll|}
\hline Group protocol of Creese et al. [11] \\
\hline $1 . \quad \forall A \longrightarrow_{N} \forall A^{\prime} \quad: A, P k_{A}, T_{A}$ \\
2. $\quad \forall A \longrightarrow_{N} \forall A^{\prime} \quad:$ all Messages $\left.1, N_{A}\right\}_{P k_{A^{\prime}}}$ \\
3a. $A$ displays $\quad:$ shorthash $\left(\left\{\right.\right.$ all Messages $2^{d}$ \}), number of processes \\
3b. $\quad \forall A \longrightarrow_{E} \forall A^{\prime} \quad:$ users compare hashes and check numbers \\
4. $\quad \forall A \longrightarrow_{N} \forall A^{\prime} \quad:$ longhash $\left(\left\{\right.\right.$ all Messages $2^{d}$ \}) \\
\hline
\end{tabular}

The protocol is shown in $[41,32,33]$ by Roscoe to be vulnerable to a man-in-the-middle and one-shot attack, related to the birthday paradox. The flaw arises from the short output of the shorthash function shorthash() used in Messages 3a, and the intruder's ability to manipulate the content of Messages 1 and 2. The details of the attack can also be found in [32, 33, 41]. In spite of the attack, the protocol invented in 2003 introduced implicitly the principle P1 in Section 4.3, which contributes to the optimisation of not only empirical work but also computational cost.

In summer 2005 [41], Roscoe corrected this flaw by introducing a trustworthy leader $L$ who is responsible for generating a fresh key $k_{L}$ of order $B=160$ bits that is inputted into the digest function used in this scheme. The following is a slightly simplified version of this protocol, introduced in $[32,33]$. Here, $S$ represents a typical slave node, and $A$ a typical node (either $L$ or $S$ ). $\operatorname{init}(L, A)$ is true if $L=A$ and false otherwise.

\begin{tabular}{|l}
\hline $\begin{array}{l}\text { Hash Commitment Before Knowledge } \\
\text { HCBK protocol, [32, 33, 41] }\end{array}$ \\
\hline $0 . \quad L \quad{ }_{N} \forall S \quad: L$ \\
$1 . \quad \forall A \quad{ }_{N} \forall A^{\prime} \quad:\left(A, I N F O_{A}\right)$ \\
$2 a . \quad L \quad{ }_{N} \forall S \quad:$ longhash $\left(k_{L}\right)$ \\
$2 b . \quad \forall S \quad{ }_{E} L \quad:$ committed \\
$3 . \quad L \quad{ }_{N} \forall S \quad: k_{L}$ \\
$4 . \quad \forall A \quad{ }_{E} \forall A^{\prime} \quad: \operatorname{digest}\left(k_{L}, I N F O S\right)$, init $(L, A)$ \\
\hline Computational cost: $W^{2}+N M=25+N M$ \\
\hline
\end{tabular}

In this scheme, the parties have to agree on the b-bit digest of INFOS and the leader's key $k_{L}$ over the empirical channel. In addition, Message $2 \mathrm{~b}$ has all the slaves communicate to $L$ that they have received Message 2a, and are committed to their final digest value (though none of them

\footnotetext{
${ }^{38}$ Messages 4 in this protocol are similar in purpose to Messages 4 in Hoepman (Section 4.1).
} 
know it yet). Thus the 1-bit commitment signal must be sent over the unforgeable empirical channel that cannot be blocked. We will see shortly this represents one side of an interesting trade-off. In term of computation cost, each node has to compute a single cryptographic hash of key $k_{L}$ and a $b$-bit digest value of $I N F O S$, resulting in a cost of order: $W^{2}+N M=25+N M$, thanks to the use of the cost model given in Section 2.4.2.

The protocol is termed HCBK standing for Hash Commitment Before Knowledge, and its security relies on the trustworthiness of the leader $L$ who generates the single digest key and consequently has control over the final digest value. ${ }^{39}$ We have seen one previous protocol in which one party determines the final agreed value and there are two stages of commitment/agreement from the other parties (there the single other party). That is Wong and Stajano's "one-way empirical channel" protocol [57] from Section 4.3. In fact if Messages 4 and 5 in that protocol are interchanged (a possibility we noted there), it is not hard to see that it becomes an indirect binding variant on pairwise HCBK.

In many circumstances, it is possible for such a leader to emerge (for example as the system whose owner initiates the protocol). However this is complicated if there may be an untrustworthy party present, since the leader must be trustworthy for the protocol to have any security.

In order to avoid this problem, the authors designed a protocol in which, provided the protocol has completed, any pair or a sub-group of honest parties will have obtained the authentic information of each other irrespective of what other (dishonest) parties may have done. In [32, 33] we identified the following second principle, derived from the leader's role in HCBK and the direct binding strategy of commitment before knowledge, that essentially makes parties committed to the final digest value before any of them knows what the value actually is.

P2 A protocol offers the adversary no strategy to force digest agreement to be more likely than chance if, at some point in every partial run, for some node $A$,

1. $A$ is committed to a value $d$ such as $d=\operatorname{digest}\left(k^{*}, I N F O S\right)$; and

2. $A$ has randomly selected a value $k_{A}$ such that:

(a) $k_{A}$ randomises the value of $k^{*}$;

(b) no other participant knows the value of $k_{A}$ at this point in the run; and

(c) no input received by $A$ can eliminate $A$ 's randomising effect of $k_{A}$ on $k^{*}$.

This is clearly a formalisation and refinement of the (joint) commitment before knowledge concept that we have used throughout this paper.

In the resulting protocol, every node will plays a role similar to the leader in HCBK and thus follow P2: each node $A$ now needs some fresh and unpredictable sub-key $k_{A}$ (of $B=160$ bits say) to contribute to the final digest value.

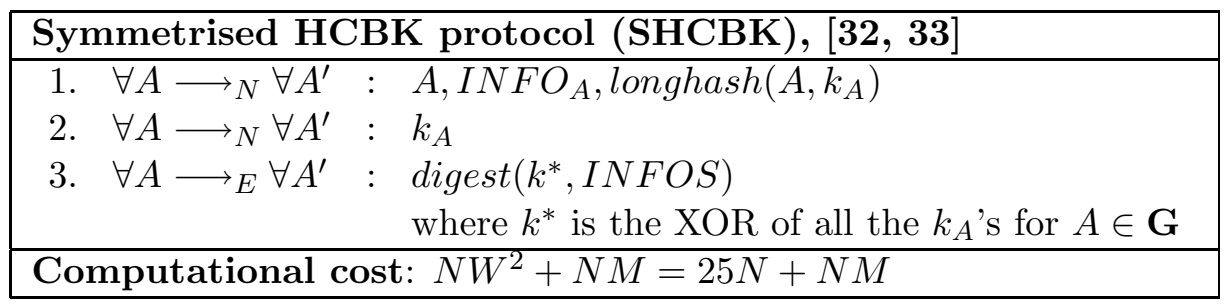

\footnotetext{
${ }^{39}$ The readers can find the full security analysis of HCBK in $[32,33]$.
} 
In the first messages, the purpose of the inclusion of the identity $A$ inside the longhash is to prevent an intruder from eliminating $A$ 's randomising effect on $k^{*}$ by simply copying its longhash value, i.e. this follows part 2(c) of principle P2 ${ }^{40}$ This protocol also eliminates the one-bit commitment signals from the slaves to $L$.

This protocol is termed Symmetrised HCBK due to the similarity with HCBK and its symmetrical structure. Since everyone takes responsibility separately for influencing the final digest key $k^{*}$ and the final digest value, neither any one nor any proper subset of $\mathbf{G}$ can determine the digest value until all the sub-keys are revealed in Messages 2. Indeed, whatever other parties do, the influence of a particular node $A$ completely randomises the final digest value. As a result, this authenticates trustworthy parties to each other irrespective of what others (dishonest nodes) may have done. In other words, this protocol is tolerant of corrupt parties: one of the main challenges in designing group protocols as mentioned at the beginning of Section 5 .

The use of XOR to combine different sub-keys in SHCBK protocol was shown to be secure in [33]. The intuitive reason behind this choice of the operator is that, thanks to the identities included in longhashes of Messages 1 to avoid a reflexive attack, both final digest keys (denoted $k_{A}^{*}$ and $k_{B}^{*}$ ) computed at trustworthy parties $A$ and $B$ are uniform random variables that can be considered independent of all $k_{C}^{*}$ introduced by other parties (corrupt or otherwise). $k_{A}^{*}$ and $k_{B}^{*}$ can either be independent or dependent. When they are independent of each other, the probability of a digest anomaly is $2^{-b}$ as defined in the first part of the digest specification given in Section 2.2.2. When they are dependent (which they will be - indeed equal - if all nodes are trustworthy and there is no intruder), the only relation that can occur between them is linear of the form $k_{B}^{*}=\theta \oplus k_{A}^{*}$ where the intruder can choose $\theta$, as discussed in [33]. But this again does not give him any advantage thanks to the second part (in particular " $\oplus \theta$ ") of the digest specification.

The robust security achieved here comes at the expense of increased computation cost relative to HCBK: each node now has to compute $N$ longhashes (one for generating its own Message 1 and $N-1$ for checking the coherence of what other nodes send) as opposed to the single longhash value of HCBK. The computation cost of SHCBK is thus $N W^{2}+N M=25 N+N M$. This is the other side of the trade-off mentioned above: we have gained in increased corruption tolerance and the loss of the empirical commit signal, but lost computational efficiency.

Though designed as group protocols, both HCBK and SHCBK can be easily turned into pairwise ones. If we replace the two-way empirical channels used in HCBK with one-way channels from the slaves to the leader then we will have a one-way authentication group protocol: all the slaves are authenticated to the leader.

\subsection{Indirect binding group protocol}

We have claimed that the direct binding approach (HCBK and SHCBK protocols) remains more efficient in group protocols than indirect binding as it was in pairwise ones. The argument is true because it is more efficient to have the SAS created from the presumed large INFOS rather than it is to have each $I N F O_{A}$ bound to random nonces by full-power cryptography to resist combinatorial search. In order to illustrate this advantage, we will generalise the (symmetrical) indirect binding pairwise scheme of Čagalj, Čapkun and Hubaux [6] in Section 4.2.1 into a group protocol. The level

\footnotetext{
${ }^{40}$ We note the same protection is required in Čagalj-Čapkun-Hubaux whenever there are two or more longhashes or commitments. This reflexive attack does not work against HCBK as there is only one cryptographic hash, generated by the leader, longhash $\left(k_{L}\right)$.
} 
of security achieved by this scheme is the same as SHCBK: (1) tolerant of corrupt parties; and (2) the probability of successful one-shot attack is upper bounded by $2^{-b}$, here $b$ is still the bitlength of the single SAS transmitted over the empirical channel.

\begin{tabular}{|c|c|}
\hline Indirec & -binding group protocol $\mathrm{New}$ \\
\hline 1. $\forall A$ & $\longrightarrow_{N} \forall A^{\prime}: I N F O_{A}, c_{A}$ \\
\hline & Where $c_{A} \| d_{A}=\operatorname{commit}\left(A, I N F O_{A}, R_{A}\right)$, \\
\hline & $R_{A}$ is randomly picked by $A$. \\
\hline 2. $\forall A$ & $\longrightarrow_{N} \forall A^{\prime}: d_{A}$ \\
\hline & $A^{\prime}$ computes $R_{A}=\operatorname{open}\left(A, I N F O_{A}, c_{A}, d_{A}\right)$ \\
\hline 3. $\forall A$ & $\longrightarrow_{E} \forall A^{\prime}: \bigoplus_{A \in \mathbf{G}} R_{A}$ \\
\hline Compu & ational cost: $N W M=5 N M$ \\
\hline
\end{tabular}

From the protocol, we can see that all of the $I N F O_{A}$ s must be committed separately: each node always has to commit once (for its own INFO), and de-commit or open $(N-1)$ times to verify the commitments of all other parties. This results in a computation cost of order $N W M=5 N M$, which is approximately $W=5$ times as expensive as either HCBK or SHCBK.

An important observation we want to make is that in this scheme any untrustworthy party $I$ can fool other participants of group $\mathbf{G}$ into accepting different versions of its own $I N F O$, i.e. $I N F O_{I}$ and $I N F O_{I}^{\prime}$. This can be easily done if $I$ sends the commitments of different versions of its $I N F O$ relative to the same short random nonce $R_{I}$ to others in the first messages.

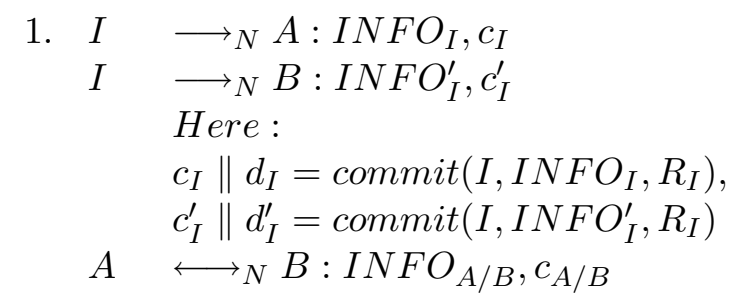

2. $I \longrightarrow{ }_{N} A: d_{I}$

$I \quad \longrightarrow_{N} B: d_{I}^{\prime}$

$A \longleftrightarrow{ }_{N} B: d_{A / B}$

3. $\forall A \longrightarrow{ }_{E} \forall A^{\prime}: R_{I} \oplus R_{A} \oplus R_{B}$

Thus parties still agree the XOR of all short nonces manually in the third messages. However, we do not consider this as a valid attack because we do not care whether we get the right or wrong information about an untrustworthy node in an authentication protocol. Conversely, if we want to turn this into a key agreement protocol then the first assumption we have to make is that all participants are honest, as discussed at the start of this section.

\subsection{Modified versions of HCBK and SHCBK}

The difference in efficiency between SHCBK and HCBK raises the question of whether it is possible to reduce the amount of computation processing in SHCBK without compromising its security, i.e. being tolerant of corrupt parties and the probability of a successful one-shot attack is bounded by $2^{-b}$. A small improvement turns out to be possible if we make use of a technique used in Vaudenay's one-way scheme and direct binding pairwise protocols in Sections 4.2.1 and 4.3. On the one hand, 
this can slightly reduce the number of commitments or longhashes at each node. On the other hand, it makes the schemes asymmetrical in structure. This will be explained as follows.

Let us assume there are $(N-1)$ leaders $L$ out of a total of $N$ parties, where each leader has to generate a fresh sub-key, compute and send its longhash over the normal network. The single node left is the unique slave $S$, who transmits its fresh sub-key $k_{S}$ to other nodes after receiving longhashes from every leader. Below, $A$ is a typical node which is either $S$ or $L$.

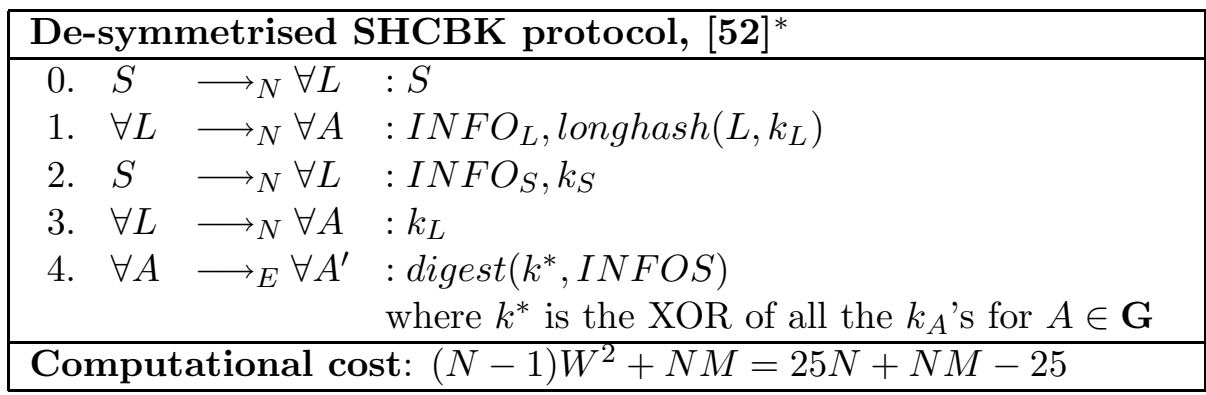

We discovered this shortly after SHCBK. It was also independently invented by Valkonen, Asokan and Nyberg [52], who were not aware of SHCBK and neither addressed the issue of tolerance of untrustworthy parties nor the use of a digest function.

As can be seen from the protocol, while there is no commitment attached to the sub-key $k_{S}$ of the slave, the fact that it is the only one treated in this way guarantees that it will not be manipulated by the intruder. This is as resistant to corrupt participants as SHCBK, but of course separate arguments are required in considering a pair of trustworthy ones, depending on whether one of them is the single slave or not.

At the expense of introducing the role of the slave and making the protocol asymmetrical, the total number of longhashes per node declines to $(N-1)$ which corresponds to a processing cost of $(N-1) W^{2}+N M=25 N+N M-25$. This is cheaper than SHCBK by $W^{2}=25$ units per node, though we suspect that the asymmetry introduced into the communication regime will in practice mean that it is no better: nodes will spend more time waiting. Nevertheless, it illustrates the possibility of further improving the computation efficiency by careful analysis.

Unfortunately, it appears impossible to employ the same technique to decrease the number of longhashes further. Once there are two or more slaves in a single run, the scheme will be vulnerable to a man-in-the-middle attack in which the intruder impersonates all the slaves to talk to all the leaders and vice versa. Intuitively, this is because principle $\mathbf{P 2}$ has been violated: any slave, who sends its $k_{A}$ before having $k_{B}$ (or a commitment like longhash $\left(k_{B}\right)$ ) for each other $B$, is revealing its last piece of information too soon before it is committed to the final digest value. An example of this attack, applied to the case of one leader and two slaves, can be demonstrated in Appendix B.

We can however reduce computational cost if we are prepared to weaken our corruption tolerance requirement towards that of $\mathrm{HCBK}$. With the addition of 1-bit empirical commitment signals like those in HCBK and allowing the number of leaders $l$ to vary between 1 and $N$, we propose a hybrid protocol. In other words, rather than having a single leader generating the digest key by itself as in HCBK, we will now have $l$ leaders generating $l$ sub-keys, here $l \in[2, N]$. The effect is that all of the leaders would have to be corrupt for the protocol to fail, otherwise the probability of a successful one-shot attack is upper bounded by $2^{-b}$. For example, if everyone trusts $A$ or $B$, then it may be appropriate to choose both as leaders, meaning that all nodes have to compute $l=2$ longhashes.

Below, $S$ represents a slave, $L$ is a leader, and $A$ is either a slave or a leader. $S L$ is the set 
of $l$ leaders' identities broadcasted to every one in Message 0 by a single node $T$, who knows this information.

\begin{tabular}{|c|c|}
\hline \multicolumn{2}{|c|}{ Hybrid HCBK New } \\
\hline $\begin{array}{ll}0 . & T\end{array}$ & $\longrightarrow_{N} \forall A: S L$ \\
\hline 1. $\forall A$ & $\longrightarrow_{N} \forall A^{\prime}: A, I N F O_{A}$ \\
\hline 2a. $\forall L$ & $\longrightarrow_{N} \forall A: \operatorname{longhash}\left(L, k_{L}\right)$ \\
\hline 2b. $\forall S$ & $\longrightarrow{ }_{E} \forall L: 1$-bit committed signal \\
\hline 3. $\forall L$ & $\longrightarrow{ }_{N} \forall A: k_{L}$ \\
\hline 4. $\forall A$ & $\longrightarrow{ }_{E} \forall A^{\prime}: \operatorname{digest}\left(k^{*}, I N F O S\right)$, leader $(S L, A)$ \\
\hline & Where $k^{*}$ is the XOR of all the $k_{L}$ 's for $L \in S L$ \\
\hline
\end{tabular}

The protocol is termed the Hybrid Hash Commitment Before Knowledge (HHCBK) protocol because it applies to the hybrid case and is in effect a hybrid of HCBK and SHCBK.

One problem here is establishing which of the nodes are to be leaders in such a way that this does not add greatly to the empirical communication burden of the protocol.

Let us assume that the set $S L$ of leaders is actually established by insecure communications between the nodes. One way to make the protocol secure would be to have all nodes agree not only the digest but also the set of leaders with each other and with their systems' views on this subject: we assume that leader $(S L, A)$ indicates whether $A$ is a leader or not. Post hoc this establishes agreement on who the leaders are a very strong way, but with a lot of leaders it could be expensive.

Imagine a weaker rule: a leader has no duty to check on the leader information from others, and a slave only has to convince himself that there is, amongst leaders who announce themselves, at least one leader who is trustworthy. This is perhaps surprisingly sufficient.

To see this note that slaves $A$ and $B$, and a trustworthy leader $L$ (amongst those identified by $A$ ) have all agreed the digest. We should consider a number of possibilities, all of which could be brought about by the intruder and the weaker use of the leader information.

- $A$ 's final digest was not influenced by sub-key $k_{L}$. In this case, the probability of $A$ 's and $L$ 's digests agreeing is no more than $2^{-b}$, by $\mathbf{P 2}$ applied to $L$.

- A's final digest was influenced by sub-key $k_{L}$, as was $B$ 's. In this case, we can use the same argument that applies to HCBK.

- $A$ 's final digest was influenced by $k_{L}$, but $B$ 's was not. In this case, final digest keys $k_{A}^{*}$ and $k_{B}^{*}$ are independent.

Of course, in order for the digests to agree, it is necessary that the nodes as opposed to their human users know who all the leaders are. What the argument above shows is that it is not always necessary for the humans to check every detail of this.

It is interesting to see the trade-off between the preliminary security assumption and the computation cost of $l W^{2}+N M=25 l+N M$ in this protocol. What this formula tells us is if we want to improve the computation cost of the protocol, we need to decrease the number of leaders $l$ in the group $\mathbf{G}$, and in effect increasing the trustworthiness requirement from each leader. 


\section{Conclusions and further work}

In this section, we tabulate efficiency analysis of the various protocols discussed in this paper, discuss the results and other topics relevant to these classes of protocol as well as looking ahead to work that still needs to be done.

\subsection{Efficiency}

In this section, we tabulate the efficiency of all the protocols we have described according to the two measures we have used throughout: the amount of empirical communication and the computation effort required for the cryptographic primitives. More complex models might take into account the amount of high bandwidth required and a measure of the concurrency that is possible between nodes, but we do not go into that level of detail.

We group them into three tables: non-interactive (one-way) authentication, interactive mutual authentication and group protocols.

Our main measure of empirical work is the number of bits that each user has to compare. Of course we do not imagine that they will compare actual bits, but some more friendly representation of the data! There is also a trade-off between how much work it is to compare information and the degree of certainty we have that human users will actually do the work required of them. At one extreme we can imagine the leader in an HCBK network announcing the final digest and asking the rest of the humans present to put up their hand if the value displayed on their PDA's does not agree; at the other we can imagine that an implementation allowing the connection of a credit card to a merchant might require the customer to type the merchant's digest into his card (or a device holding it) so the card can do the comparison itself. But both these last issues are implementation dependent and orthogonal to the logical structure of the underlying protocol, so we will stick to our simple measure. In those protocols that require the extra confirmation message over the empirical channel (MANA I, Wong-Stajano, HCBK, HHCBK etc) we write " $b+1$ " as the amount of empirical effort.

In these tables we have used the simple cost model of hash and digest functions described in Section 2.4.2: the cost is proportional to the product of the length of the information being digested and the width of the output.

The non-interactive protocols are shown in Table 2. There is a relationship between how much we assume of the empirical channel and how much work is required over it. Unlike later tables, we might note that the levels of security are not the identical in the protocols listed: here $B=160$ and $B / 2=80$ are examples of the numbers of bits required to make a hash function strongly and weakly collision resistant, it is assumed that $2^{-b}=2^{-16}$ likelihood of one-shot attacker success is sufficiently small in all cases (except the first two protocols), but for reasons discussed earlier (V-)MANA I does not attain this. Of course, one might want to change any of these numbers for good reason, but we believe that the relative differences of them will not be greatly different if this is done. Therefore, the lessons about relative cost that this table teaches us will remain true.

The same will, naturally, be true of the other tables. The reader is advised to regard constants like $160=B, 32=w, 16=b$ and $25=(160 / 32)^{2}=(B / w)^{2}=W^{2}$ as "variable constants", where exact numbers are given for illustrative purposes.

The other tables cover pairwise protocols and groups. For the latter, in each case we get another pairwise protocol by setting $N=2$ : these are all competitive in the pairwise table. 
It is also necessary to point that since most direct binding protocols invented by other authors to date do not use a digest function to produce SASs, we will therefore illustrate the difference by giving the computation cost of both cases in 3 tables. The truncated longhash or universal hash functions ( [49] that require a longhash to compress large messages into a fixed number of bits initially) will be denoted (longhash).

Thanks to the principle $\mathbf{P} \mathbf{1}$ and the use of the digest function, in general direct binding protocols are much more efficient than indirect binding ones as can be seen from all three tables: about up to $B / w=W=5$ times more efficient should $M$ gets large. ${ }^{41}$ The larger $M$ (the length of $I N F O S$ ) is, the more accurate this effect. ${ }^{42}$ This is likely to be the case whenever

(i) A large amount of authenticated information is being passed from one participant to another. We might note in this connection that direct binding protocols are a more efficient way of doing this than any method that the nodes are likely to use once a secure connection is up and running, since the latter is likely to use either conventional symmetric cryptography or standard length hashes.

(ii) Large amounts of information needs to be passed to enable the users of the network to be able to associate the logical members of the network either to other human users (e.g. photographs) or function (e.g. manufacturer's certificate).

(iii) There are many nodes present in a group: INFOS can be expected to expand proportionately to this.

With respect to group protocols we recall that there is a trade-off between processing cost and the amount of corruption resistance required as well as with eliminating the 1-bit confirmation message.

\subsection{Short-term public key cryptography}

We anticipate that in many, probably a majority, of the practical uses of the classes of protocol described in paper, one of the main objectives is the bootstrapping of a means of secret and authenticated communication between the parties. In almost all such cases, we expect that this will be done by establishing a symmetric session key to be used in conjunction with some encryption algorithm in a way that gives both secrecy and authentication.

One cannot establish such a session key directly in the $I N F O_{A} \mathrm{~s}$, since all such information is public following the protocol run. Rather, as anticipated in many of the protocols and discussion earlier in this paper, we can expect that this is done either by including public keys in the $I N F O_{A} \mathrm{~s}$, or alternatively Diffie-Hellman tokens. Of course Diffie-Hellman tokens can then be combined directly into session keys, whereas public keys have to be used properly to establish authenticated session keys.

\footnotetext{
${ }^{41}$ In Section 2.4.2 the digest output length is rounded up to 1 word (32 bits). However if we have a 16 bit digest and a 8/16 bit processor (which will often be the case in lightweight devices) then the advantage of digest over hashing grows (in other words direct over indirect bindings), potentially, to 10 times $=\frac{5 \mathrm{~W}}{0.5 \mathrm{~W}}$ (from 5 ).

${ }^{42}$ This is not necessarily a clear advantage for direct binding in the case of the Hoepman and Wong-Stajano protocols because the information parties want to have authenticated only includes one or two Diffie-Hellman tokens that are quite small.
} 


\begin{tabular}{|l|c|c|c|}
\hline Protocol & Binding & $\begin{array}{c}\text { Human } \\
\text { work }(\text { bit })\end{array}$ & Computation cost \\
\hline Balfanz et al. & Direct & $B=160\left(\rightarrow_{W E}\right)$ & $W M=5 M$ \\
\hline Pasini-Vaudenay & Indirect & $B / 2=80\left(\rightarrow_{W E}\right)$ & $\frac{M W}{2}+\frac{W^{2}}{4}=2.5 M+6.25$ \\
\hline Mashatan-Stinson & Direct & $B / 2=80\left(\rightarrow_{W E}\right)$ & $(M+W / 2) W / 2=2.5 M+6.25$ \\
\hline MANA I (CBC-MAC) & Direct & $2 b+1=33\left(\rightarrow_{E}\right)$ & $W M=5 M$ \\
\hline V-MANA I $($ digest $)$ & Direct & $2 b=32\left(\rightarrow_{S E}^{t}\right)$ & $M$ \\
\hline Improved MANA I & Direct & $b+1=17\left(\rightarrow_{E}^{t}\right)$ & $M+W^{2}=M+25$ \\
\hline Improved MANA I & Indirect & $b+1=17\left(\rightarrow_{E}^{t}\right)$ & $W(M+W)=5 M+25$ \\
\hline Improved MANA I & Direct(D-H) & $b+1=17\left(\rightarrow_{E}^{t}\right)$ & $W M+M=6 M$ \\
\hline Improved V-MANA I & Direct & $b=16\left(\rightarrow_{B E}^{t}\right)$ & $M+W{ }^{2}=M+25$ \\
\hline Improved V-MANA I & Indirect & $b=16\left(\rightarrow_{B E}^{t}\right)$ & $W(M+W)=5 M+25$ \\
\hline Improved V-MANA I & Direct(D-H) & $b=16\left(\rightarrow_{B E}^{t}\right)$ & $W M+M=6 M$ \\
\hline
\end{tabular}

Table 2: One-way authentication protocols

\begin{tabular}{|l|c|c|c|}
\hline Protocol & Binding & $\begin{array}{c}\text { Human } \\
\text { work(bit) }\end{array}$ & Computation cost \\
\hline Hoepman & Direct & $2 b=32$ & $2(W M+M)=12 M$ \\
\hline Improved Hoepman & Direct & $b=16$ & $W M+M=6 M$ \\
\hline $\begin{array}{l}\text { Improved Hoepman' } \\
\text { (one-way empirical) }\end{array}$ & Direct & $b+1=17$ & $W M+M=6 M$ \\
\hline $\begin{array}{l}\text { Wong-Stajano } \\
\text { (one-way empirical) }\end{array}$ & Direct & $b+1=17$ & $W(2 M+1)=10 M+5$ \\
\hline Wong-Stajano & Indirect & $2 b=32$ & $2 W(2 M+W)=20 M+50$ \\
\hline Improved Wong-Stajano & Indirect & $b=16$ & $2 W(M+1)=10 M+10$ \\
\hline Vaudenay $\rightarrow W E)$ & Indirect & $b=16$ & $W M=5 M$ \\
\hline Cagalj-Capkun-Hubaux & Indirect & $b=16$ & $2 W M=10 M$ \\
\hline Pasini-Vaudenay (longhash) & Hybrid & $b=16$ & $W M+W M=10 M$ \\
\hline Pasini-Vaudenay (digest) & Hybrid & $b=16$ & $W M+M=6 M$ \\
\hline Bluetooth 2 $($ longhash $)$ & Direct & $b=16$ & $W(2 M+W)+2 W(M+W)=20 M+75$ \\
\hline Bluetooth $2($ digest $)$ & Direct & $b=16$ & $W(2 M+W)+2 M=12 M+25$ \\
\hline Laur-Nyberg (longhash) & Direct & $b=16$ & $W^{2}+2 W M=10 M+25$ \\
\hline Laur-Nyberg (digest) & Direct & $b=16$ & $W{ }^{2}+2 M=2 M+25$ \\
\hline Vaudenay-style (digest) & Direct & $b=16$ & $W^{2}+2 M=2 M+25$ \\
\hline
\end{tabular}

Table 3: Interactive pairwise two-way authentication protocols (unless indicated they all use twoway empirical channels: ${ }_{E}$ ) 


\begin{tabular}{|c|c|c|c|}
\hline Protocol & Binding & $\begin{array}{c}\text { Human } \\
\text { work(bit) }\end{array}$ & Computation cost \\
\hline Indirect binding & Indirect & 16 & $W N M=5 N M$ \\
\hline HCBK & Direct & $b+1=17$ & $N M+W^{2}=N M+25$ \\
\hline SHCBK & Direct & $b=16$ & $N M+N W^{2}=N M+25 N$ \\
\hline De-symmetrised & & & \\
SHCBK(longhash) & Direct & $b=16$ & $W N M+W^{2}(N-1)=5 N M+25(N-1)$ \\
\hline $\begin{array}{c}\text { De-symmetrised } \\
\text { SHCBK (digest) }\end{array}$ & Direct & $b=16$ & $N M+W^{2}(N-1)=N M+25(N-1)$ \\
\hline Hybrid HCBK & Direct & $b+1=17$ & $N M+W^{2} l=N M+25 l$ \\
\hline
\end{tabular}

Table 4: Group authentication protocols (they all use empirical channels: $\longrightarrow_{E}$ )

In our environment where we desire low power consumption and perhaps simple processors, the large modulus calculations needed to perform either Diffie-Hellman or public-key cryptography are unattractive. It is worth noting however that there are opportunities for efficiencies in the use of public keys arising from the style in which we use them.

In a PKI, it is public keys themselves that are used for long-term authentication. Any breach of such a key will have disastrous long-term consequences. However, in our usage, public keys can be fresh for every run of a protocol and are only used once or twice in the initial set-up phase. So provided we can be confident that a public key cannot be broken during the length of a session, we can be sure that the communication in that session are properly authenticated, and that any computing power directed at cryptanalysing it subsequently can only reveal the secrets of a single session.

The generation of fresh public/private key pairs can, or course, be done in advance of a session or a collection of them might be "loaded" periodically onto a device that does not have the computational power to generate them. (This would, naturally, have to be from a trusted source perhaps it is even an extra function built into the device's power supply!)

In any event, a security assessment of a particular application may well, because of the shortterm nature of public keys, require shorter (and therefore easier to use) public keys than in a PKI.

\subsection{Conclusions}

It this paper we have surveyed the literature on a new and - we believe - important style of protocol, examining non-interactive, interactive and group protocols. We have also discovered that, even though groups of these protocols have been invented independently and presented in different notations, the basic principle of commitment before knowledge underlies all of those that either attain or nearly attain the optimal empirical performance.

Very different from any other families of security or cryptography protocols, human interaction plays a central and very important role in the security of the authentication schemes presented in this survey. For this reason, we have tried to rigorously analyse how much human effort (measured in the number of bits the humans have to keep in their minds) is required, and more importantly whether it is optimal with respect to the obtained level of security. And we are glad to claim that 
the result has been very positive in all three types of authentication protocols.

On the one hand, our aim of this survey is to summarise and categorise all existing protocols invented so far into comprehensive groups. On the other hand, we also try to give the readers a better of view of where this research area is heading to, and what can be done to make these protocols usable in practice.

\subsection{Future research}

After running a successful session of one of the group protocols, the group has essentially bootstrapped a local PKI. If such a local PKI is going to be more than short term, we are going to have to address issues such as how to add extra nodes, form the union of two groups, and excluding nodes. In other words how does one maintain a local PKI? An initial, but somewhat inefficient, approach to this is described in [52].

The nature of the protocols we have described, and especially the need to take the combinatorial search power of attackers into account when quantifying security, apparently fall outside the range of the successful tools for protocol analysis produced in the last decade or so. If this new class of protocols is to be as important as we believe it is important either that these tools or their methodologies are developed or new tools created to handle them. It may well be appropriate to use or adapt probabilistic model checkers such as PRISM [1] for this purpose.

Another interesting possibility is to apply and extend our existing work in authentication protocols in pervasive computing into other security applications such as electronic polling/voting (physical envelopes) [31], auction protocols (anonymous physical broadcast channel) [48] and e-cash [8] where human interaction is also employed but little if any investigation has been undertaken to analytically quantify and optimise them.

And finally, designing efficient ways of comparing the SAS manually in different circumstances (and applications) are also very important for the future of these protocols. As a result, this area has received much attention from many different research groups [16, 25, 28, 29, 45, 51] recently.

\section{Acknowledgements}

Long Nguyen's work on this paper was supported by studentships from QinetiQ Trusted Information Management and the Ministry of Education and Training of Vietnam.

Roscoe's work on this paper was partially supported by funding from the US Office of Naval Research.

We are grateful to anonymous referees whose detailed comments allowed us to greatly improve the paper.

\section{References}

[1] See: http://www.prismmodelchecker.org/

[2] Simple Pairing White Paper. See:

www.bluetooth.com/NR/rdonlyres/

OA0B3F36-D15F-4470-85A6-F2CCFA26F70F/0/

SimplePairing_WP_V10r00.pdf 
[3] D. Balfanz, D. Smetters, P. Stewart and H. Wong. Talking to strangers: Authentication in Ad Hoc Wireless Networks. In Symposium on Network and Distributed Systems Security, 2002.

[4] M. Bellare and P. Rogaway. Entity Authentication and Key Distribution. Advances in Cryptology - Crypto 1993, LNCS vol. 773, pp. 232-249.

[5] J. Bierbrauer, T. Johansson, G.A. Kabatianskii and B.J.M. Smeets. On Families of Hash Functions via Geometric Codes and Concatenation. Advances in Cryptology - Crypto 1993, LNCS vol. 773, pp. 331-342.

[6] M. Čagalj, S. Čapkun and J. Hubaux. Key agreement in peer-to-peer wireless networks. Proceedings of the IEEE Special Issue on Security and Cryptography, vol. 94, no. 2, pp. 467-478, 2006 .

[7] J.L. Carter and M.N. Wegman. Universal Classes of Hash Functions. Journal of Computer and System Sciences, vol. 18 (1979), pp. 143-154.

[8] D. Chaum. Secret-ballot receipts: True voter-verifiable elections. Security and Privacy Magazine, IEEE, Jan.-Feb. 2004. Volume 2, Issue: 1, pp. 38-47.

[9] S.J. Creese, M.H. Goldsmith, R. Harrison, A.W. Roscoe, P. Whittaker and I. Zakiuddin. Exploiting empirical engagement in authentication protocol design. In Proceedings of the International Conference on Security in Pervasive Computing $\left(S P C^{\prime} 05\right)$, LNCS vol. 3450, pp. 119-133, 2005.

[10] S.J. Creese, M.H. Goldsmith, A.W. Roscoe and M. Xiao. Bootstrapping multi-party ad-hoc security. In Proceedings of IEEE Security Track, pp. 369-375, 2006.

[11] S.J. Creese, M.H. Goldsmith, A.W. Roscoe and I. Zakiuddin. The attacker in ubiquitous computing environments: Formalising the threat model. Workshop on Formal Aspects in Security and Trust, 2003. IIT-CNR Technical Report.

[12] S.J. Creese, M.H. Goldsmith, A.W. Roscoe and I. Zakiuddin. Security properties and mechanisms in human-centric computing. Proceedings of Workshop on Security and Privacy in Pervasive Computing, 2004.

[13] C. Gehrmann, C. Mitchell and K. Nyberg. Manual Authentication for Wireless Devices. RSA Cryptobytes, vol. 7, no. 1, pp. 29-37, 2004.

[14] C. Gehrmann and K. Nyberg. Security in personal area networks. In C. J. Mitchell, editor, Security for Mobility, pp. 191-230. IEE, London, 2004.

[15] International Organisation for Standardisation, Geneve, Switzerland. ISO/IEC 9798 Information technology - Security techniques - Entity authentication - Part 6: Mechanisms using manual data transfer, 2003.

[16] M.T. Goodrich, M. Sirivianos, J. Solis, G. Tsudik and E. Uzun. Loud and Clear: HumanVerifiable Authentication Based on Audio. IEEE International Conference on Distributed Computing Systems, (ICDCS'06), pp. 10-33. 
[17] J.-H. Hoepman. Ephemeral Pairing on Anonymous Networks. In D. Hutter and M. Ullmann, editors, International Conference on Security in Pervasive Computing, SPC 2005, LNCS vol. 3450, pp. 101-116.

[18] J.-H. Hoepman. Ephemeral Pairing Problem. International Conference Financial Cryptography, LNCS vol. 3110, pp. 212-226, 2004.

[19] G.A. Kabatianskii, B. Smeets and T. Johansson. On the cardinality of systematic authentication codes via error-correcting codes. IEEE Transactions on Information Theory, IT-42 (1996), pp. 566-578.

[20] H. Krawczyk. LFSR-based Hashing and Authentication. Advances in Cryptology - Crypto 1994, LNCS vol. 839, pp. 129-139.

[21] H. Krawczyk. New Hash Functions For Message Authentication. Advances in Cryptology Eurocrypt 1995, LNCS vol. 921, pp. 301-310.

[22] S. Laur and K. Nyberg. Efficient Mutual Data Authentication Using Manually Authenticated Strings. LNCS vol. 4301 on LNSC, pp. 90-107, 2006.

[23] S. Laur, N. Asokan and K. Nyberg. Efficient mutual data authentication using manually authenticated strings: Extended version. Cryptology ePrint Archive, Report 2005/424, 2006.

[24] A.Y. Lindell Comparison-Based Key Exchange and the Security of the Numeric Comparison Mode in Bluetooth v2.1. Topics in Cryptology CT-RSA, LNCS vol. 5473, pp. 66-83, 2009.

[25] A. Madhavapeddy, D. Scott, R. Sharp and E. Upton. Using Camera Phones to Enhance Human-Computer Interaction. Proceedings of Ubiquitous Computing (UbiComp 2004), pp. $1-2,2004$.

[26] Y. Mansour, N. Nisan and P. Tiwari. The Computational Complexity of Universal Hashing. Proceedings of the 22nd Annual ACM Symposium on Theory of Computing, pp. 235-243, 1990.

[27] A. Mashatan and D.R. Stinson. Non-interactive two-channel message authentication based on hybrid-collision resistant hash functions. IET Information Security, 2007, vol. 1 (3), pp. 111118.

[28] R. Mayrhofer and M. Welch. A Human-Verifiable Authentication Protocol Using Visible Laser Light. International Conference on Availability, Reliability and Security. ARES 2007, pp. 11431148 .

[29] J.M. McCune, A. Perrig and M.K. Reiter. Seeing is Believing: Using Camera Phones for Human-Verifiable Authentication. IEEE Symposium on Security and Privacy, pp. 110-124, 8-11 May 2005.

[30] A.J. Menezes, P.C. van Oorschot and S.A. Vanstone. Handbook of Applied Cryptography. ISBN: 0-8493-8523-7.

[31] T. Moran and M. Naor. Polling with Physical Envelopes: A Rigorous Analysis of a HumanCentric Protocol. Advances in Cryptology - Eurocrypt 2006, LNCS vol. 4004, pp. 88-108. 
[32] L.H. Nguyen and A.W. Roscoe. Efficient group authentication protocol based on human interaction. Proceedings of Workshop on Foundation of Computer Security and Automated Reasoning Protocol Security Analysis, pp. 9-31, 2006.

[33] L.H. Nguyen and A.W. Roscoe. Authenticating ad hoc networks by comparison of short digests. Information and Computation, vol. 206 (2008), pp. 250-271. Special Issue of Information and Computation on Computer Security: Foundations and Automated Reasoning 2006.

[34] L.H. Nguyen and A.W. Roscoe. New combinatorial bounds for universal families of hash functions. Manuscript is available. A short version of the paper is presented at the Summer School on Provable Security, 2009. See: http://www.comlab.ox.ac.uk/files/749/uhf .pdf

[35] L.H. Nguyen and A.W. Roscoe. Separating two roles of hashing in one-way message authentication. Proceedings of FCS-ARSPA-WITS 2008, pp. 195-210.

[36] L.H. Nguyen and A.W. Roscoe. Efficient digest function based on Toeplitz matrix and integer multiplication, in preparation.

[37] L.H. Nguyen. First committee draft of ISO/IEC 9798-6 - Entity authentication using manual data transfers. This was submitted to the committee of ISO/IEC JTC1/SC 27 (working group $2)$.

[38] S. Pasini and S. Vaudenay. SAS-based Authenticated Key Agreement. Public Key Cryptography - PKC 2006: The 9th international workshop on theory and practice in public key cryptography, LNCS vol. 3958, pp. 395-409.

[39] S. Pasini and S. Vaudenay. An Optimal Non-interactive Message Authentication Protocol. Topics in Cryptology - CT-RSA 2006: The Cryptographers' Track at the RSA Conference 2006, LNCS vol. 3860, pp. 280-294.

[40] R. Pass. On Deniability in the Common Reference String and Random Oracle Model. Advances in Cryptology - Crypto 2003, LNCS vol. 2729, pp. 316-337.

[41] A.W. Roscoe. Human-centred computer security. See: http://web.comlab.ox.ac.uk/oucl/work/bill.roscoe/ publications/113.pdf, 2005.

[42] A.W. Roscoe and L.H. Nguyen. Security in computing networks. Published by the World Intellectual Property Organization (WIPO). Publication Number: WO/2007/052045. Publication date: 10.05.2007. International Application No.: PCT/GB2006/004113. See: http://www.wipo.int/pctdb/en/ wo.$j$ sp? wo $=2007052045 \& I A=W 02007052045 \& D I S P L A Y=S T A T U S$

[43] A.W. Roscoe, B. Chen and L.H. Nguyen. Improvements in communications security. International Patent Application No. PCT/GB07/004963, published by the World Intellectual Property Organization (WIPO), publication number: WO/2008/078101, publication date: 03.07.2008.

[44] A.W. Roscoe and L.H. Nguyen. Improvements related to the authentication of messages. Priority patent application number 0811210.4, filed on 18 June 2008. 
[45] N. Saxena, J.-E. Ekberg, K. Kostiainen and N. Asokan. Secure Device Pairing based on a Visual Channel. In the Proceedings of the IEEE Symposium on Security and Privacy 2006, pp. 306-313.

[46] N. Smart. Cryptography, An Introduction. ISBN $0007709987 \quad 7 \quad$ (PB). Or see: http://en.wikipedia.org/wiki/Chosen_plaintext_attack

[47] F. Stajano and R. Anderson. The resurrecting duckling: Security issues for ad-hoc wireless networks. Workshop on Security Protocols 1999, LNCS vol. 1976, pp. 172-194.

[48] F. Stajano and R. Anderson. The Cocaine Auction Protocol: on the Power of Anonymous Broadcast. In the Proceedings of the 3rd International Workshop on Information Hiding, LNCS, 1999.

[49] D.R. Stinson. Universal Hashing and Authentication Codes. Advances in Cryptology - Crypto 1991, LNCS vol. 576, pp. 74-85, 1992.

[50] J. Suomalainen, J. Valkonen and N. Asokan. Security Associations in Personal Networks: A Comparative Analysis. In the Proceedings of the 4th European Workshop on Security and Privacy in Ad hoc and Sensor Networks 2007. LNCS vol. 4572, pp. 43-57.

[51] E. Uzun, K. Karvonen and N. Asonka. Usability Analysis of Secure Pairing Methods. Nokia Research Center, Technical Report NRC-TR-2007-002, January 2007. In the Usable Security (USEC '07) workshop 2007. LNCS vol. 4886, pp. 307-324, 2008.

[52] J. Valkonen, N. Asokan and K. Nyberg. Ad Hoc Security Associations for Groups. In Proceedings of the Third European Workshop on Security and Privacy in Ad hoc and Sensor Networks 2006. LNCS vol. 4357, pp. 150-164.

[53] S. Vaudenay. Secure Communications over Insecure Channels Based on Short Authenticated Strings. Advances in Cryptology - Crypto 2005, LNCS vol. 3621, pp. 309-326.

[54] M.N. Wegman and J.L. Carter. New Hash Functions and Their Use in Authentication and Set Equality. Journal of Computer and System Sciences, vol. 22, pp. 265-279, 1981.

[55] A.F. Webster and S.E. Tavares. On the Design of S-Boxes. Advances in Cryptology 1985, LNCS vol. 218, pp. 523-534, 1986.

[56] Ford-Long Wong and F. Stajano. Multi-channel Protocols. Proceedings of the 13th International Workshop on Security Protocols 2005. LNCS vol. 4631, pp. 128-132.

[57] Ford-Long Wong and F. Stajano. Multi-channel Security Protocols. IEEE Pervasive Computing, vol. 6 (4), pp. 31-39, Oct-Dec 2007.

\section{A The importance of empirical display of leader $(L, A)$ in Hybrid HCBK}

In order to illustrate that if the information leader $(L, A)$ were not be communicated over the empirical channel, the protocol would suffer from an attack, we shall look at the situation where 
there are two users $A$ and $B$. The intruder invents $I N F O_{X}^{\prime}$ for each of them in which it says $X$ is a leader ${ }^{43}$. In fact neither $A$ nor $B$ act as a leader, and the intruder is able to send hash keys to $A$ and $B$ such that the final digests agree. So they agree on the final digest, each believing the others to be the leader. Of course this works equally well with any two disjoint sets of "leaders". This attack works when we can block the commitment sent via empirical channel. Otherwise both $A$ and $B$ will realise something wrong going on as both of them are not supposed to receive any commitment as neither of them created any longhash. This will depend on whether commitment signals are directed at only specific leaders in Message $2 b$, which is specified in this protocol to save the amount of human work, however real life implementations might vary significantly from our specification.

\section{B Attack on group protocol with two slaves}

In this appendix, we demonstrate why the "De-symmetrised SHCBK" protocol of Section 5.3 cannot be weakened further to have two slaves, even when all the nodes in the protocol are trustworthy.

Assume that there is a leader $L$ trying to authenticate its information $I N F O_{L}$ to two slaves $A$ and $B$. In the first run $\alpha$ of the protocol, the intruder $I$ impersonates slaves $A$ and $B$ to communicate with the leader $L$, and comes up with two random keys $k_{A}^{\prime}$ and $k_{B}^{\prime}$.

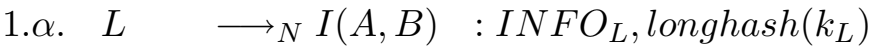

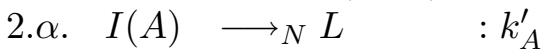

$$
\begin{aligned}
& I(B) \longrightarrow_{N} L \quad: k_{B}^{\prime}
\end{aligned}
$$

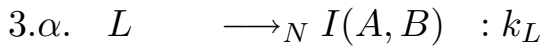

After $L$ sends out its own key $k_{L}$, the intruder can determine the final digest value of run $\alpha$ that $L$ is going to compare over the empirical network in Message 4. Let us assume that $k_{S}=k_{A}^{\prime} \oplus k_{B}^{\prime}$. To fool slaves $A$ and $B$ into thinking that a fake $I N F O_{L}^{\prime}$ is authentic, the intruder needs to find $k_{S}^{\prime}$ such that the digests of both runs come out to be the same:

$$
\operatorname{digest}\left(k_{L} \oplus k_{S}, I N F O_{L}\right)=\operatorname{digest}\left(k_{L} \oplus k_{S}^{\prime}, I N F O_{L}^{\prime}\right)
$$

This should not take a long time as the bitlength of the digest output is short. Once he successfully searches for $k_{S}^{\prime}$, he starts the second run $\beta$. In this run, he impersonates the leader $L$ to talk to slaves $A$ and $B$ as well as modifying the their keys as follows

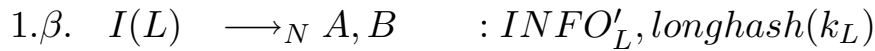

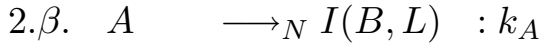

$$
\begin{aligned}
& B \quad \longrightarrow_{N} I(A, L): k_{B} \\
& I(A) \quad \longrightarrow_{N} B \quad: k_{B} \oplus k_{S}^{\prime} \\
& I(B) \longrightarrow_{N} A \quad: k_{A} \oplus k_{S}^{\prime} \\
& \text { 3. } \beta . \quad I(L) \longrightarrow_{N} A, B \quad: k_{L}
\end{aligned}
$$

After the key $k_{L}$ is revealed to $A$ and $B$, all three nodes should be able to empirically agree on two equal digests that have different antecedents. In other words, the slaves accept $I N F O_{L}^{\prime}$ faked by the intruder.

\footnotetext{
${ }^{43} A$ will receives $I N F O_{B}^{\prime}$ saying that $B$ is the leader and vice versa.
} 


$$
\begin{aligned}
& \text { 4. } \beta . \quad A, B \quad \longrightarrow_{E} L \quad: \operatorname{digest}\left(k_{L} \oplus k_{S}^{\prime}, I N F O_{L}^{\prime}\right) \\
& A \quad{ }_{E} B \quad: \operatorname{digest}\left(k_{L} \oplus k_{S}^{\prime}, I N F O_{L}^{\prime}\right)
\end{aligned}
$$

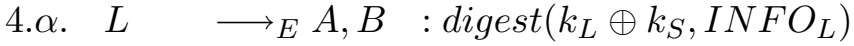

The digests of all three nodes will agree despite them not agreeing on $I N F O_{L}$.

Note that not only does the above attack work when we $X O R$ digest subkeys as in SHCBK protocol, but also with any other ways to combine them. This is because the intruder will always be able to predetermine the final digest value before any slave is committed to the digest. Since a digest value is short, it is feasible for the intruder to search for digest subkeys that map to the digest value regardless of how nodes choose to combine them.

\section{Improved protocols of MANA I and their security analysis}

In this Appendix, we will present another two versions of Improved MANA I, which are termed the indirect binding and Diffie-Hellman style (or D-H style) protocols.

\section{C.1 Indirect binding and D-H style versions of Improved MANA I}

An alternative solution for Improved V-MANA I is to use a commitment scheme to bind $I N F O_{A}$ to a $b$-bit random nonce $R$, which is generated by $A$ and released over the bounded empirical channel. This therefore makes use of the indirect information binding strategy, as can be seen below.

\begin{tabular}{|cll|}
\hline \multicolumn{2}{|c|}{ Improved version of V-MANA I (indirect binding) [35] } \\
\hline $1 . \quad A \longrightarrow N$ & $B: I N F O_{A}, c$ \\
& & $(c, d)=\operatorname{commit}\left(I N F O_{A}, R\right)$ \\
2. $A \longrightarrow{ }_{B E}^{t}$ & $B: R$ \\
$3 . \quad A \longrightarrow{ }_{N}$ & $B: d$ \\
\hline Computational cost: $W(M+W)=5 M+25$ \\
\hline
\end{tabular}

The order and time constraints of messages' arrival in this scheme must be the same as in the direct binding version of Improved V-MANA I. However, this protocol is expensive to run because the large $I N F O_{A}$ must be processed by a long output commitment scheme, which is more expensive than a digest function: $W(M+W)=25+5 M$, i.e. an approximate $(W=5)$-fold increase compared to the direct binding version.

It is interesting to note that this protocol might be regarded as the non-interactive version of the pairwise (indirect binding) protocol of Vaudenay [53] of Section 4.2.

Similar to the direct binding version of Improved MANA I, we can replace the bounded delay empirical channel with a simple acknowledgement to have the following scheme.

\begin{tabular}{|ccl|}
\hline \multicolumn{2}{|c|}{ Improved version of MANA I (indirect binding) [35] } \\
\hline $1 a$. & $A \longrightarrow N$ & $B: I N F O_{A}, c$ \\
& & $(c, d)=\operatorname{commit}\left(I N F O_{A}, R\right)$ \\
$1 b$. & $B \longrightarrow$ & $A: 1$-bit committed signal \\
2. & $A \longrightarrow E$ & $B: R$ \\
3. & $A \longrightarrow N$ & $B: d$
\end{tabular}


Next we describe another improved scheme, whose main idea is taken root from the pairwise (direct binding) authentication protocol of Hoepman [17, 18] in Section 4.1.

In the following description, $k$ is a long secret key (160-bit) of $A$ that corresponds to his DiffieHellman token $g^{k}$ he wants to authenticate. In order for the following protocol to be secure, the Diffie-Hellman token $g^{k}$ must be fresh at each session, unpredictable and kept secret to $A$ when its longhash and $b$-bit shorthash are revealed in the first two messages.

\begin{tabular}{|ll|}
\hline \multicolumn{2}{|c|}{ Improved version of V-MANA I (D-H style) [35] } \\
\hline $1 . A \longrightarrow_{N} \quad B:$ longhash $\left(g^{k}\right)$ \\
2. $A \longrightarrow_{B E}^{t} \quad B:$ shorthash $\left(g^{k}\right)$ \\
3. $A \longrightarrow_{N} \quad B: g^{k}$ \\
\hline Computational cost: $W M+M=6 M$ \\
\hline
\end{tabular}

The main difference between this and the direct/indirect binding versions is that there is no $I N F O_{A}$ sent in Message 1 because the Diffie-Hellman token, revealed in Message 3, plays the dual-role of both $I_{N F O}$ and the long secret key. This results in a cost of order $W M+M=6 M$.

\section{C.2 Security analysis of the Improved (V-)MANA I protocols}

We will adapt the Bellare-Rogaway security model where an intruder can control on which node a new protocol instance is launched, and so we are going to define the two kinds of adversaries used our security analysis.

1. A general adversary can launch multiple instances of participants ( $A$ and $B$ in our protocols). As commonly the case in the literature, the number of times that (s)he can launch an instance of any participant is limited by a finite number, for example $\mathcal{Q}_{A}$ for $A$ and $\mathcal{Q}_{B}$ for $B$. The time complexity of this adversary is bounded by a finite number say $T$. This is the kind of adversary we want to prove our protocols resist in the security analysis presented here.

2. A one-shot adversary is a special case of the general adversary where the number of each participant's instances he can launch is at most once, in other words, $\mathcal{Q}_{A}=\mathcal{Q}_{B}=1$.

We are going to prove that the Improved (V-)MANA I protocols are secure against a one-shot attack in the first step, and then use Theorem 1 stated below to lift the one-shot attack's model to a general attack's model.

The following theorem is the combined result of Lemma 6 of Vaudenay [53] and Theorem 5 of Pasini and Vaudenay [39].

Theorem $1[35,39,53]$ We consider a general attack such that the number of instances of $A$ (respectively $B$ ) is at most $\mathcal{Q}_{A}$ (respectively $\mathcal{Q}_{B}$ ).

If there exists a one-shot attack against the three improved versions of the (V-)MANA I protocol which has success probability $p$ in a time $T$, then a general attack is successful with probability $P \leq p \cdot \mathcal{Q}_{A}$ in a time $Q_{A} T$.

In the following and all subsequent security proofs, we only consider the case when the intruder cannot influence random keys and nonces which are generated by $A$ 's instances (possibly launched 
by the intruder) and which are instrumental in the computation of SASs. ${ }^{44}$ Note, we believe that the same assumption has also been made by Vaudenay in his proof of this theorem (i.e. Lemma 6 of [53]).

Proof An instance of $A$ is compatible with an instance of $B$ if $B$ 's instance succeeded and received all messages in the right order, where Message 2 is transmitted over the empirical channels from the corresponding $A$ 's instance.

The number of possible compatible pairs of instances is upper bounded by $\mathcal{Q}_{A} \mathcal{Q}_{B}$, which can be reduced to $\mathcal{Q}_{A}$ in the Improved (V-)MANA I protocols because

- In the Improved versions of MANA I, the single SAS (i.e. digest or random nonce) transmitted over empirical channels by definition in Section 2.1 cannot be mistaken, replayed or delayed from one to another session.

- In the Improved versions of V-MANA I, $B$ can always be offline. As a result, the intruder can simulate all instances of $B$ and picks one who will make the attack succeed.

When an attack is successful, there should exist one compatible pair of instances of $A$ and $B$ which (1) have or compute the same SAS value sent over the empirical channel; and (2) do not share the same public data $I N F O_{A}$ that they try to agree on.

Note, the SASs' values of all compatible pairs of instances are uniformly distributed and independent ${ }^{45}$ from one another because the SASs are randomised by either random keys ( $k$ in direct binding), random nonces ( $R$ in indirect binding), or random Diffie-Hellman tokens $\left(g^{k}\right.$ in the DiffieHellman style version). All of these random elements, which are instrumental in the computation of SASs, are unknown to the intruder at the point when they were generated by $A$ 's instances thanks to the above assumption. (This argument remains true even when data $I N F O_{A}$ s are controlled by the intruder in the direct binding version, thanks to the use of digest functions).

We know that the probability of a successful attack on each compatible pair of instances is limited to $p$ in a time $T$ (i.e. $A$ and $B$ agree on the same digest of different preimage data $I N F O_{A} \mathrm{~s}$ ). We therefore have that the general adversary is successful with probability $P \leq p \cdot \mathcal{Q}_{A}$ in a time $Q_{A} T$.

\section{C.2.1 Security analysis of the direct binding improved (V-)MANA I}

In the following theorem, the notation $\left(\epsilon_{c}, T_{c}\right)$-collision-resistant indicates that the success probability of finding a hash collision is upper bounded by $\epsilon_{c}$ in a time $T_{c}$. Similarly, $\left(\epsilon_{i}, T_{i}\right)$-inversionresistant indicates that the success probability of inverting a hash value is upper bounded by $\epsilon_{i}$ in a $T_{i}$.

Theorem 2 [35] Given that longhash () is $\left(\epsilon_{c}, T_{c}\right)$-collision-resistant and $\left(\epsilon_{i}, T_{i}\right)$-inversion-resistant, a general attack with number of $A$ 's (respectively $B$ 's) instances bounded by $\mathcal{Q}_{A}$ (respectively $\mathcal{Q}_{B}$ ) is successful against the direct binding versions of Improved (V-)MANA with probability $2^{-b} \mathcal{Q}_{A}\left(1+\epsilon_{i}+\epsilon_{c}\right)$ in a time $Q_{A}\left(T_{i}+T_{c}\right)$.

\footnotetext{
${ }^{44}$ The assumption must be made even though the intruder can launch new instances of any party or device, for otherwise, the intruder could easily fool $B$ into accepting a fake $I N F O_{A}^{\prime}$ by searching for a digest or short hash collision. Examples are long key $k$ in the direct binding version of Improved (V-)MANA I, and short nonce $R$ and commitment value $c$ in the indirect binding ones.

${ }^{45}$ See Footnote 12 for what independence means.
} 
The following proof applies to the direct binding version of Improved V-MANA I, but it can be slightly modified to cope with the direct binding version of Improved MANA I.

Proof We first find the probability of a successful one-shot attack.

A one-shot intruder has no advantage of sending fake $I N F O_{A}^{\prime}$ and $\operatorname{longhash}\left(k^{\prime}\right)$ to $B$ (masquerading as $A$ ) after the digest is released in Message 2. Therefore, after $I N F O_{A}$ and longhash $(k)$ are sent in Message 1 where $k$ is a private, fresh and long (160-bit) key generated by $A$ in each session and is unknown to any one including the intruder, there are three possibilities that can happen: ${ }^{46}$ (1) with probability $\epsilon_{c}$ the intruder can find a hash collision in a time $T_{c}$; (2) with probability $\epsilon_{i}$ the intruder can invert the hash value in a time $T_{i}$; and (3) with probability $\left(1-\epsilon_{c}-\epsilon_{i}\right)$ neither can the intruder find a hash collision nor invert the hash value. Note, there is no need to consider the 2nd-preimage resistance property of a hash function since the intruder does not know key $k$ generated by the honest party $A$ in Message 1 .

1. With probability $\epsilon_{c}$ in a time $T_{c}$, the adversary can search (off-line) for two distinct keys $k^{\prime}$ and $k^{\prime \prime}$ for which longhash $\left(k^{\prime}\right)=$ longhash $\left(k^{\prime \prime}\right)$. The adversary then sends an arbitrarily data $I N F O_{A}^{\prime}\left(I N F O_{A}^{\prime} \neq I N F O_{A}\right)$ and longhash( $\left.k^{\prime}\right)$ to $B$ (masquerading as $A$ ).

\begin{tabular}{|lll|}
\hline \multicolumn{2}{|c|}{ Game against the improved V-MANA I (direct binding)- hash collision } \\
\hline $1 . \quad A \quad \longrightarrow N \quad I(B): I N F O_{A}$, longhash $(k)$ \\
& $I(A) \longrightarrow{ }_{N} \quad B \quad: I N F O_{A}^{\prime}$, longhash $\left(k^{\prime}\right)$ \\
$2 . \quad A \quad \longrightarrow S E \quad B \quad: \operatorname{digest}\left(k, I N F O_{A}\right)$ \\
\hline $3 . \quad A \quad \longrightarrow N \quad I(B) \quad: k$ \\
\hline Winning condition: digest $\left(k, I N F O_{A}\right)=\operatorname{digest}\left(k^{\prime}, I N F O_{A}^{\prime}\right)$ or \\
digest $\left(k, I N F O_{A}\right)=\operatorname{digest}\left(k^{\prime \prime}, I N F O_{A}^{\prime}\right)$
\end{tabular}

Prior to sending a key to $B$ in Message 3 the adversary checks to see whether or not $\operatorname{digest}\left(k, I N F O_{A}\right)=\operatorname{digest}\left(k^{\prime}, I N F O_{A}^{\prime}\right)$, and/or $\operatorname{digest}\left(k, I N F O_{A}\right)=\operatorname{digest}\left(k^{\prime \prime}, I N F O_{A}^{\prime}\right)$. In the first case (which has probability $2^{-b}$ ), the adversary sends $k^{\prime}$ to $B$. In the second case (which also has probability $2^{-b}$ ), the adversary sends $k^{\prime \prime}$ to $B$. We conclude that a one-shot attack has probability $2 \epsilon_{c} 2^{-b}$ of success in a time $T_{c}$.

2. With probability $\epsilon_{i}$ in a time $T_{i}$, the adversary can find a preimage $k^{\prime}$ such that longhash $\left(k^{\prime}\right)=$ longhash $(k)$. The adversary then replaces $I N F O_{A}$ with an arbitrarily data $I N F O_{A}^{\prime}\left(I N F O_{A}^{\prime} \neq\right.$ $\left.I N F O_{A}\right)$ in Message 1.

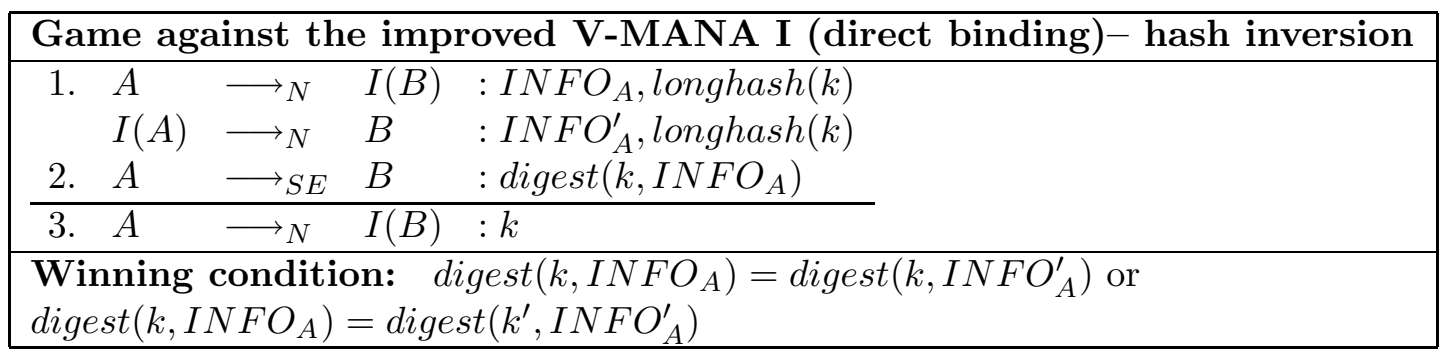

\footnotetext{
${ }^{46}$ We assume that given any $I N F O_{A}$ and longhash $(k)$, it is infeasible to gain any advantage in predicting the value of $\operatorname{digest}\left(k, I N F O_{A}\right)$, i.e. the digest value should be uniformly distributed even in the presence of $m$ and longhash $(k)$.
} 
Prior to sending a key to $B$ the adversary checks to see whether or not $\operatorname{digest}\left(k, I N F O_{A}\right)=$ $\operatorname{digest}\left(k, I N F O_{A}^{\prime}\right)$, and/or $\operatorname{digest}\left(k, I N F O_{A}\right)=\operatorname{digest}\left(k^{\prime}, I N F O_{A}^{\prime}\right)$. Similar to the previous case, a one-shot attack has probability $2 \epsilon_{i} 2^{-b}$ of success in a time $T_{i}$.

3. On the other hand, with probability $\left(1-\epsilon_{i}-\epsilon_{c}\right)$ in a time $\left(T_{i}+T_{c}\right)$ neither can the adversary search for a hash collision or invert the hash value. Thus the adversary has to select a random pair $\left(k^{\prime}, I N F O_{A}^{\prime}\right)$ where $I N F O_{A} \neq I N F O_{A}^{\prime}$.

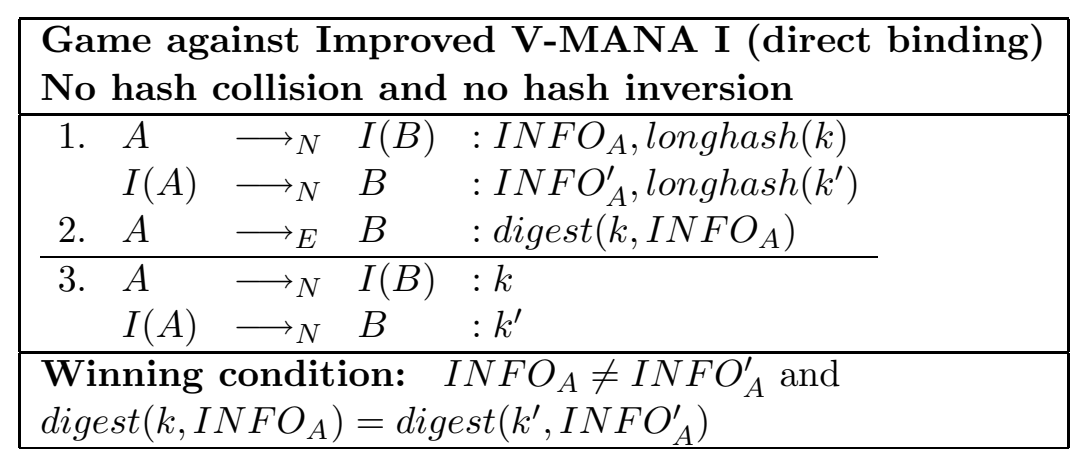

Clearly, the probability of success of this case is $\left(1-\epsilon_{i}-\epsilon_{c}\right) 2^{-b}$ in a time $\left(T_{i}+T_{c}\right)$ thanks to the digest specification.

We conclude that any one-shot adversary in a time $\left(T_{i}+T_{c}\right)$ has the following probability of success

$$
p \leq 2 \epsilon_{c} 2^{-b}+2 \epsilon_{i} 2^{-b}+\left(1-\epsilon_{c}-\epsilon_{i}\right) 2^{-b}=2^{-b}\left(1+\epsilon_{c}+\epsilon_{i}\right)
$$

We now can apply Theorem 1 to deduce that any general adversary has probability $2^{-b} \mathcal{Q}_{A}\left(1+\epsilon_{c}+\epsilon_{i}\right)$ of success in a time $Q_{A}\left(T_{i}+T_{c}\right)$.

\section{C.2.2 Security analysis of the indirect binding improved (V-)MANA I}

Theorem 3 [35] Given that a commitment scheme is $\left(\epsilon_{h}, T_{h}\right)$-hiding and $\left(\epsilon_{b}, T_{b}\right)$-binding, a general attack with number of $A$ 's (respectively $B$ 's) instances bounded by $\mathcal{Q}_{A}$ (respectively $\mathcal{Q}_{B}$ ) is successful against the indirect binding versions of Improved (V-)MANA with probability $\left(\epsilon_{h}+\epsilon_{b}\right) \mathcal{Q}_{A}$ in a time $Q_{A}\left(T_{b}+T_{h}\right)$.

The following proof gives supporting evidence for the security of the indirect binding version of Improved (V-)MANA I.

Proof There are two possibilities that a one-shot attacker can do after receiving $I N F O$ and $c$ in Message 1 from $A$ :

- Leaving $c$ unchanged, the intruder sends $I N F O_{A}^{\prime}$ and $c$ to $B$ (masquerading as $A$ ) where $I N F O_{A}^{\prime} \neq I N F O_{A}$. With probability $\epsilon_{b}$ in a time $T_{b}$, the intruder can come up with a $d^{\prime}$ (which can be either the same as or different from $d$ revealed in Message 3) such that $\operatorname{open}\left(I N F O_{A}^{\prime}, c, d^{\prime}\right)=R$ thanks to the binding property of a commitment scheme. 
- With probability $\epsilon_{h}$ in a time $T_{h}$, the intruder can guess the value of $R$ from $I N F O_{A}$ and $c$, and then compute $\left(c^{\prime}, d^{\prime}\right)$ such that open $\left(I N F O_{A}^{\prime}, c^{\prime}, d^{\prime}\right)=R$ thanks to the hiding property of a commitment scheme. ${ }^{47}$

We can apply Theorem 1 to deduce that any general intruder has a success probability $\mathcal{Q}_{A}\left(\epsilon_{b}+\epsilon_{h}\right)$ in a time $Q_{A}\left(T_{h}+T_{b}\right)$.

\section{C.2.3 Security analysis of Improved V-MANA I in Diffie-Hellman style}

Theorem 4 [35] Given that longhash () is $\left(\epsilon_{c}, T_{c}\right)$-collision-resistant and $\left(\epsilon_{i}, T_{i}\right)$-inversion-resistant, a general attack with number of $A$ 's (respectively $B$ 's) instances bounded by $\mathcal{Q}_{A}$ (respectively $\mathcal{Q}_{B}$ ) is successful against the Improved V-MANA I protocol in Diffie-Hellman (D-H) style with probability $2^{-b} \mathcal{Q}_{A}\left(1+\epsilon_{c}\right)$ in a time $Q_{A}\left(T_{c}+T_{i}\right)$.

Proof As in the proof of Theorem 2, there are three possibilities which can happen after $A$ releases Message $1:^{48}$

1. With probability $\epsilon_{c}$ in a time $T_{c}$, the adversary can search for two distinct D-H tokens $g^{k^{\prime}}$ and $g^{k^{\prime \prime}}$ for which longhash $\left(g^{k^{\prime}}\right)=\operatorname{longhash}\left(g^{k^{\prime \prime}}\right)$. The adversary then sends longhash $\left(g^{k^{\prime}}\right)$ to $B$ (masquerading as $A$ ).

\begin{tabular}{|l}
\hline \multicolumn{2}{|c|}{ Game against the improved V-MANA I (D-H style)- hash collision } \\
\hline $1 . \quad A \quad \longrightarrow N \quad I(B):$ longhash $\left(g^{k}\right)$ \\
$I(A) \longrightarrow N \quad B \quad:$ longhash $\left(g^{k^{\prime}}\right)$ \\
$2 . \quad A \quad \longrightarrow S E \quad B \quad: \operatorname{shorthash}\left(g^{k}\right)$ \\
\hline $3 . \quad A \quad \longrightarrow N \quad I(B): g^{k}$ \\
\hline Winning condition: $\operatorname{shorthash}\left(g^{k}\right)=\operatorname{shorthash}\left(g^{k^{\prime}}\right)$ or \\
shorthash $\left(g^{k}\right)=\operatorname{shorthash}\left(g^{k^{\prime \prime}}\right)$
\end{tabular}

A one-shot attack has probability $2 \epsilon_{h} 2^{-b}$ of success in a time $T_{c}$.

2. With probability $\epsilon_{i}$ in a time $T_{i}$, the adversary can find a preimage $g^{k^{\prime}}$ such that longhash $\left(g^{k}\right)=$ longhash $\left(g^{k^{\prime}}\right)$. The adversary then replaces $g^{k}$ with $g^{k^{\prime}}$ in Message 3 and hopes that they produce the same $b$-bit hash output. Therefore, the probability of success is $\epsilon_{i} 2^{-b}$ in a time $T_{i}$.

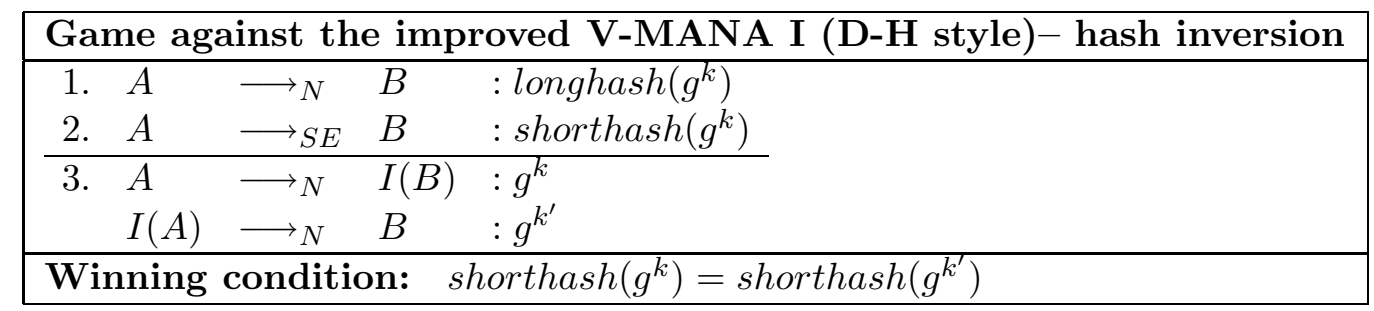

\footnotetext{
${ }^{47}$ Since $I N F O_{A} \neq I N F O_{A}^{\prime}$, it is very unlikely that $c=c^{\prime}$.

${ }^{48}$ We also assume that given longhash $\left(g^{k}\right)$ it is infeasible for the intruder to gain any advantage in predicting the value of $\operatorname{shorthash}\left(g^{k}\right)$.
} 
3. On the other hand, with probability $\left(1-\epsilon_{i}-\epsilon_{c}\right)$ in a time $T_{i}+T_{c}$ neither can the adversary search for a hash collision or invert the hash value. Thus the adversary has to select a random D-H token $g^{k^{\prime}}$ and send longhash $\left(g^{k^{\prime}}\right)$ to $B$ in Message 1 (masquerading as $A$ ).

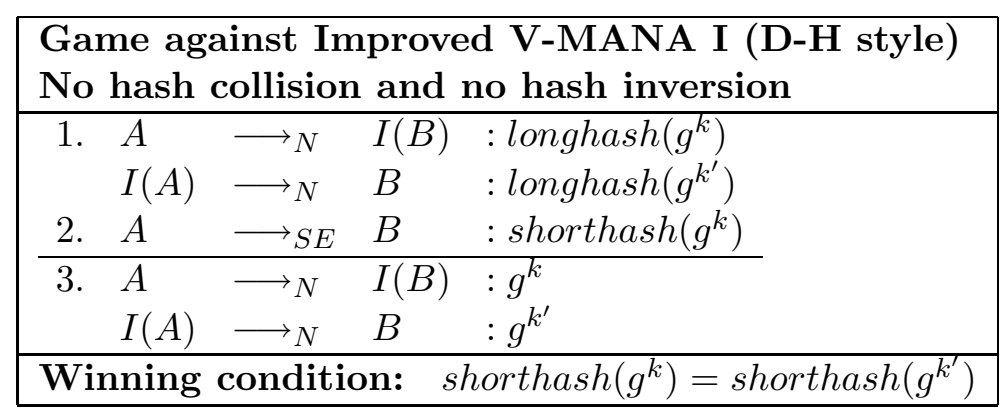

Clearly, the probability of success of this case is $\left(1-\epsilon_{i}-\epsilon_{c}\right) 2^{-b}$.

We conclude that any one-shot adversary in a time $T_{i}+T_{c}$ has the following probability of success

$$
p \leq 2 \epsilon_{c} 2^{-b}+\epsilon_{i} 2^{-b}+\left(1-\epsilon_{c}-\epsilon_{i}\right) 2^{-b}=2^{-b}\left(1+\epsilon_{c}\right)
$$

We now can apply Theorem 1 to deduce that any general adversary has a success probability $2^{-b} \mathcal{Q}_{A}\left(1+\epsilon_{c}\right)$ in a time $Q_{A}\left(T_{i}+T_{c}\right)$. 